\title{
Formal deformations and their categorical general fibre
}

\author{
Daniel Huybrechts, Emanuele Macrì, and Paolo Stellari
}

\begin{abstract}
We study the general fibre of a formal deformation over the formal disk of a projective variety from the view point of abelian and derived categories. The abelian category of coherent sheaves of the general fibre is constructed directly from the formal deformation and is shown to be linear over the field of Laurent series. The various candidates for the derived category of the general fibre are compared.

If the variety is a surface with trivial canonical bundle, we show that the derived category of the general fibre is again a linear triangulated category with a Serre functor given by the square of the shift functor. The paper is a companion to [9], where the results are applied to Fourier-Mukai equivalences of K3 surfaces.
\end{abstract}

Mathematics Subject Classification (2010). 18E30, 14D15.

Keywords. Derived categories, deformations, $K$-trivial surfaces.

\section{Introduction}

Let $\pi: \mathcal{X} \rightarrow \operatorname{Spf}(\mathbb{C}[[t]])$ be a formal deformation of a smooth complex projective variety $X$ given by an inductive system of flat morphisms $\pi_{n}: X_{n} \rightarrow \operatorname{Spec}\left(\mathbb{C}[t] /\left(t^{n+1}\right)\right)$ with $X_{0}=X$ and isomorphisms $X_{n+1} \times_{\mathbb{C}[t] /\left(t^{n+2}\right)} \operatorname{Spec}\left(\mathbb{C}[t] /\left(t^{n+1}\right)\right) \simeq X_{n}$ over $\mathbb{C}[t] /\left(t^{n+1}\right)$. Thus, $\mathcal{X}$ as a ringed space is the topological space $X$ with $\mathcal{O}_{X}:=\lim \mathcal{O}_{X_{n}}$ as its structure sheaf. Interesting examples arise as formal neighborhoods of an actual deformation of $X$ over a smooth one-dimensional base, which may be algebraic or just a complex disk.

In order to understand the generic behavior of certain classes of varieties, it is often necessary to study the general fibre of formal deformations of the type $\pi: \mathcal{X} \rightarrow \operatorname{Spf}(\mathbb{C}[[t]])$. If the deformation is given as the formal neighbourhood of an algebraic deformation of $X$ over a curve, then the usual concept of the schemetheoretical general fibre yields a variety defined over the function field of the curve. For arbitrary formal deformations, e.g. obtained as formal neighborhoods of deformations in non-algebraic directions, a geometric construction of the general fibre as a rigid analytic variety is provided by the work of Raynaud [15]. 
The aim of this paper is to present a categorical approach to the general fibre. We construct the abelian category of coherent sheaves on the general fibre directly without first passing to the rigid analytic variety representing it geometrically. This simplifies going back and forth from sheaves on the original variety $X$ or its formal deformation $\mathcal{X}$ to sheaves on the general fibre. The passage from the abelian category to its derived category, which plays a central role in the applications we have in mind, is more difficult. Here we have to address subtle points related to Verdier quotients of triangulated categories.

To appreciate the results of this paper, we should briefly explain the main application we developed in [9]. For a smooth projective K3 surface $X$, Orlov proved in [14] that any autoequivalence of the bounded derived category of coherent sheaves $\mathrm{D}^{\mathrm{b}}(X)$ induces an isomorphism of the total cohomology group $H^{*}(X, \mathbb{Z})$ preserving a natural weight-2 Hodge structure and the lattice structure induced by the cup-product. In particular, there exists a homomorphism of groups $\rho$ between the group of autoequivalences $\operatorname{Aut}\left(\mathrm{D}^{\mathrm{b}}(X)\right)$ and some orthogonal group (denoted by $\mathrm{O}(\widetilde{H}(X, \mathbb{Z}))$ ) of the total cohomology group of $X$ (see [9], [14]).

Despite this nice result, a description of the image of $\rho$ had been missing for some time. In [18], Szendrôi proposed a conjecture saying that $\rho$ should send an equivalence to an isometry in $\mathrm{O}(\tilde{H}(X, \mathbb{Z}))$ with the additional property that the orientation of some 4-dimensional positive definite subspace of $H^{*}(X, \mathbb{R})$ is preserved.

In [9], we gave a positive answer to this conjecture using a deformation argument whose main steps are the following. Given a smooth projective K3 surface $X$, we study a very special formal deformation of $X$ based on its hyperkähler geometry. At this point, we argue that the derived category of the general fibre of such a deformation, despite being $\mathbb{C}((t))$-linear and not $\mathbb{C}$-linear, has the same basic features as the derived category of a generic complex analytic K3 surface (i.e. a K3 surface with trivial Picard group). The same conjecture has been solved in [8] for those surfaces. Hence, by the special choice of the deformation, we can conclude that it holds true for $X$ as well.

The main result of this paper (Theorem 1.1 below) establishes some of the fundamental properties of the derived category of the general fibre which are needed in the above strategy.

To state precisely this result, we first define the abelian and the derived category of the general fibre of a formal deformation $\pi: \mathcal{X} \rightarrow \operatorname{Spf}(\mathbb{C}[[t]])$ of $X$. Let $\operatorname{Coh}(\mathcal{X})_{0} \subset$ $\operatorname{Coh}(\mathcal{X})$ be the full abelian subcategory of coherent sheaves on $\mathcal{X}$ which are torsion over $\mathbb{C}[[t]]$, i.e. the full subcategory of all sheaves $E \in \mathbf{C o h}(\mathcal{X})$ supported on some $\mathcal{X}_{n}$, for $n \gg 0$. With this definition, $\operatorname{Coh}(\mathcal{X})_{0}$ is a Serre subcategory and the quotient category

$$
\operatorname{Coh}\left(X_{K}\right):=\operatorname{Coh}(X) / \operatorname{Coh}(\mathcal{X})_{0}
$$

is called the abelian category of coherent sheaves on the general fibre. Here, $K$ denotes the quotient field of $\mathbb{C}[[t]]$, i.e. the field of all Laurent series. One can indeed show that $\operatorname{Coh}\left(\mathcal{X}_{K}\right)$ is a $K$-linear abelian category. 
Next we denote by $\mathrm{D}^{\mathrm{b}}(\mathcal{X}):=\mathrm{D}_{\text {coh }}^{\mathrm{b}}\left(\mathcal{O}_{X}\right.$-Mod $)$ the bounded derived category of the abelian category of $\mathcal{O}_{x}$-modules with coherent cohomology. This category has a full triangulated subcategory

$$
\mathrm{D}_{0}^{\mathrm{b}}(\mathcal{X}) \subset \mathrm{D}^{\mathrm{b}}(\mathcal{X})
$$

consisting of all complexes with cohomology in $\operatorname{Coh}(X)_{0}$. The Verdier quotient

$$
\mathrm{D}^{\mathrm{b}}\left(\mathcal{X}_{K}\right):=\mathrm{D}^{\mathrm{b}}(\mathcal{X}) / \mathrm{D}_{0}^{\mathrm{b}}(\mathcal{X})
$$

is the derived category of the general fibre of the formal deformation

$$
\pi: X \rightarrow \operatorname{Spf}(\mathbb{C}[[t]]) .
$$

The fundamental properties of the derived category of the general fibre for smooth projective surfaces with trivial canonical bundle are explained in the following theorem which is the main result of the paper.

Theorem 1.1. Let $\mathcal{X} \rightarrow \operatorname{Spf}(\mathbb{C}[[t]])$ be a formal deformation of a smooth projective surface $X$ with trivial canonical bundle. The derived category of the general fibre $\mathrm{D}^{\mathrm{b}}\left(\mathcal{X}_{K}\right)$ is a $K$-linear triangulated category and the square of the shift functor defines a Serre functor. Moreover, there exists an exact $K$-linear equivalence $\mathrm{D}^{\mathrm{b}}\left(\mathcal{X}_{K}\right) \simeq$ $\mathrm{D}^{\mathrm{b}}\left(\operatorname{Coh}\left(\mathcal{X}_{K}\right)\right)$.

The latter property, which is proved in Proposition 3.10, is extensively used in [9]. Both interpretations of the derived category of the general fibre, as the Verdier quotient of triangulated categories and as the bounded derived category of the abelian category of coherent sheaves on the general fibre, are used. E.g. in [8] all autoequivalences (and in particular spherical twists) of $\mathrm{D}^{\mathrm{b}}(\mathbf{C o h}(X))$ are described for a generic (nonprojective) K3 surface $X$. The arguments apply as well to $\mathrm{D}^{\mathrm{b}}\left(\mathbf{C o h}\left(\mathcal{X}_{K}\right)\right)$ for $\mathcal{X}_{K}$ the general fibre of a very general formal deformation of a projective $\mathrm{K} 3$ surface $X$. More precisely, we show that, up to $\operatorname{shift}, \mathrm{D}^{\mathrm{b}}\left(\mathbf{C o h}\left(\mathcal{X}_{K}\right)\right)$ contains just one spherical object (namely the image of the structure sheaf $\mathcal{O}_{x_{K}}$ ). On the other hand, in order to deform a given autoequivalence of $\mathrm{D}^{\mathrm{b}}(X)$ to an autoequivalence of the derived category of the general fibre $X_{K}$ we need to work with $\mathrm{D}^{\mathrm{b}}(X)$ and its quotient $\mathrm{D}^{\mathrm{b}}\left(\mathcal{X}_{K}\right)$. In the end we prove that the deformation of the autoequivalence of $\mathrm{D}^{\mathrm{b}}(X)$ to a Fourier-Mukai equivalence $\Phi: \mathrm{D}^{\mathrm{b}}\left(\mathcal{X}_{K}\right) \rightarrow \mathrm{D}^{\mathrm{b}}\left(\mathcal{X}_{K}^{\prime}\right)$ has kernel in the abelian category $\operatorname{Coh}\left(\left(X \times_{R} X^{\prime}\right)_{K}\right)$.

In the process of proving Theorem 1.1, we will be considering a number of related technical results. To make the overview of the paper more complete, let us mention a few of them which will be particularly relevant in [9]:

(A) The spaces of morphisms in quotient categories are often difficult to describe. However, for the natural quotients $\operatorname{Coh}(\mathcal{X}) \longrightarrow \operatorname{Coh}\left(\mathcal{X}_{K}\right)$ and $\mathrm{D}^{\mathrm{b}}(\mathcal{X}) \rightarrow \mathrm{D}^{\mathrm{b}}\left(\mathcal{X}_{K}\right)$ 
they are simply given by the tensor product with the quotient field $K$, which makes both categories $K$-linear (Propositions 2.3 and 2.9).

(B) One advantage of the categorical approach to the general fibre is that the Fourier-Mukai machinery carries over easily. For example, we prove that if the Fourier-Mukai kernel $\varepsilon_{0}$ of an equivalence $\mathrm{D}^{\mathrm{b}}(X) \stackrel{\sim}{\sim} \mathrm{D}^{\mathrm{b}}\left(X^{\prime}\right)$ deforms to a complex $\mathcal{E}$ on the product of two formal deformations $X, X^{\prime} \rightarrow \operatorname{Spf}(\mathbb{C}[[t]])$, then its restriction $\mathcal{E}_{K}$ to the general fibre defines again an equivalence $\mathrm{D}^{\mathrm{b}}\left(\mathcal{X}_{K}\right) \stackrel{\sim}{\longrightarrow} \mathrm{D}^{\mathrm{b}}\left(\mathcal{X}_{K}^{\prime}\right)$ (Corollary 2.13).

We have not attempted to develop the theory in its most general form. It would certainly be natural to study the general fibre of formal deformations over more general formal rings from a categorical perspective. Unfortunately, in that case, the results (e.g. the description of the space of morphisms) would not nearly be as nice as in the simple situation of deformations over $\mathbb{C}[[t]]$. But even the one-dimensional formal deformations studied here, should be useful in other situations, although our discussion is tailored to the application to Fourier-Mukai equivalences between K3 surfaces in [9].

The plan of the paper is as follows. In Section 2 we define the abelian and derived categories of the general fibre of a formal deformation. We study their Hom-spaces and, in Sections 2.3 and 2.4, we analyze the behavior of Fourier-Mukai transforms and Fourier-Mukai equivalences when passing to the derived categories of the general fibres.

In Section 3 we complete the proof of Theorem 1.1. As a first step, we compare the Hom-spaces and the Euler pairing on the general and special fibres of a formal deformation (Section 3.1). In Section 3.2 we describe the Serre functor of the general fibre. Finally, in Section 3.4, we restrict to the case of smooth projective surfaces with trivial canonical bundle and prove the main theorem.

Notation. Denote by $R:=\mathbb{C}[[t]]$ the ring of power series in $t$ which is a complete discrete valuation ring. Its spectrum $\operatorname{Spec}(R)$ consists of two points: The closed point $0:=(t) \in \operatorname{Spec}(R)$ with local ring $R$ and residue field $\mathbb{C}$ and the generic point $(0) \in \operatorname{Spec}(R)$ with residue field $K:=\mathbb{C}((t))$, the field of Laurent series. Moreover, we put $R_{n}:=\mathbb{C}[t] /\left(t^{n+1}\right)$ with the natural surjection $R \longrightarrow R_{n}$ defining a closed embedding $\operatorname{Spec}\left(R_{n}\right) \subset \operatorname{Spec}(R)$, which is the $n$-th infinitesimal neighbourhood of $0 \in \operatorname{Spec}(R)$. The formal scheme $\operatorname{Spf}(R)$ is then described by the increasing sequence of closed subschemes $0=\operatorname{Spec}\left(R_{0}\right) \subset \operatorname{Spec}\left(R_{1}\right) \subset \cdots \subset \operatorname{Spec}\left(R_{n}\right) \subset \cdots$. Throughout we will use the following notations for the natural inclusions $(m<n)$ :

$$
\begin{gathered}
\iota_{n}: X_{n} \hookrightarrow X \text { and } \iota:=\iota_{0}: X \hookrightarrow X ; \\
i_{m, n}: X_{m} \hookrightarrow X_{n}, \quad i_{n}:=i_{n, n+1}: X_{n} \hookrightarrow X_{n+1} \text {, and } j_{n}=i_{0, n}: X \hookrightarrow X_{n} .
\end{gathered}
$$




\section{The derived category of the general fibre}

In this section we study the basic properties of the abelian category $\operatorname{Coh}\left(\mathcal{X}_{K}\right)$ of coherent sheaves on the general fibre and of the triangulated category $\mathrm{D}^{\mathrm{b}}\left(\mathcal{X}_{K}\right)$. At the end of the section we also discuss the extension of the definition and of some interesting basic properties of Fourier-Mukai functors in the setting of formal deformations and of the derived categories of their general fibres (Corollary 2.13, see also (B) in the introduction).

The reader not familiar with the notion of quotients of abelian categories by Serre subcategories or with that of Verdier quotients is strongly encouraged to read the Appendix before proceeding with this section. For the convenience of the reader we list now the main abelian and triangulated categories which will be introduced in course of the paper. We also indicate the precise section where they are defined.

- $\mathcal{O}_{X}$-Mod: the abelian category of $\mathcal{O}_{X}$-modules (Section 2.1);

- $\operatorname{Coh}(X)$ : the abelian category of coherent sheaves on $X$ (Section 2.1);

- $\operatorname{Coh}(\mathcal{X})_{0}$ : the Serre subcategory of $\operatorname{Coh}(\mathcal{X})$ consisting of sheaves supported on some $\mathcal{X}_{n}$ (Section 2.1);

- $\operatorname{Coh}(\mathcal{X})_{\mathrm{f}}$ : the full additive category $\operatorname{Coh}(\mathcal{X})$ consisting of $\mathbb{C}[[t]]$-flat sheaves (Section 2.1);

- $\operatorname{Coh}\left(\mathcal{X}_{K}\right)$ : the quotient of the category $\operatorname{Coh}(\mathcal{X})$ by $\operatorname{Coh}(\mathcal{X})_{0}(\operatorname{Section} 2.1)$;

- $\mathrm{D}^{\mathrm{b}}\left(\mathcal{O}_{X}\right.$-Mod $)$ : the bounded derived category of the abelian category $\mathcal{O}_{X}$-Mod (Section 2.2);

- $\mathrm{D}^{\mathrm{b}}(\operatorname{Coh}(\mathcal{X}))$ : the bounded derived category of the abelian category $\operatorname{Coh}(\mathcal{X})$ (Section 2.2)

- $\mathrm{D}^{\mathrm{b}}(\mathcal{X})=\mathrm{D}_{\text {coh }}^{\mathrm{b}}\left(\mathcal{O}_{X}\right.$-Mod $)$ : the full triangulated subcategory of $\mathrm{D}^{\mathrm{b}}\left(\mathcal{O}_{X}\right.$-Mod $)$ consisting of complexes with coherent cohomology (Section 2.2);

- $\mathrm{D}_{0}^{\mathrm{b}}(\mathcal{X})=\mathrm{D}_{\operatorname{Coh}\left(X_{0}\right.}^{\mathrm{b}}\left(\mathcal{O}_{X}\right.$-Mod $)$ : the full thick triangulated subcategory of $\mathrm{D}^{\mathrm{b}}(\mathcal{X})$ consisting of complexes with cohomology in $\operatorname{Coh}(\mathcal{X})_{0}(\operatorname{Section} 2.2)$;

- $\mathrm{D}^{\mathrm{b}}\left(\mathcal{X}_{K}\right)$ : the Verdier quotient $\mathrm{D}^{\mathrm{b}}(\mathcal{X}) / \mathrm{D}_{0}^{\mathrm{b}}(\mathcal{X})($ Section 2.2$)$;

- $\mathrm{D}_{0}^{\mathrm{b}}(\operatorname{Coh}(\mathcal{X}))$ : the full thick triangulated subcategory of $\mathrm{D}^{\mathrm{b}}(\operatorname{Coh}(\mathcal{X}))$ consisting of complexes with cohomology in $\operatorname{Coh}(\mathcal{X})_{0}$ (Section 2.2);

- $\mathrm{D}_{\text {perf }}\left(\mathcal{X}_{n}\right)$ : the full triangulated subcategory of perfect complexes on $\mathcal{X}_{n}(\mathrm{Sec}-$ tion 2.2);

- $\mathrm{D}^{\mathrm{b}}\left(\mathcal{X}_{K}^{c}\right)$ : the Verdier quotient $\mathrm{D}^{\mathrm{b}}(\operatorname{Coh}(\mathcal{X})) / \mathrm{D}_{0}^{\mathrm{b}}(\operatorname{Coh}(\mathcal{X}))(\operatorname{Section} 2.2)$. 
2.1. The abelian category of the general fibre. Given a formal deformation $\pi: X \rightarrow \operatorname{Spf}(R)$ of a smooth projective variety $X$, the abelian category of all $\mathcal{O}_{X}-$ sheaves will be denoted $\mathcal{O}_{X}$-Mod. Any $\mathcal{O}_{X}$-sheaf $E$ yields an inverse system of $\mathcal{O}_{X_{n}}{ }^{-}$ sheaves $E_{n}:=\iota_{n}^{*} E$ with $\mathcal{O}_{x_{n}}$-linear transition maps $E_{n} \rightarrow i_{m, n *} E_{m}$, for $n>m$, inducing isomorphisms $i_{m, n}^{*} E_{n} \simeq E_{m}$. Then $\lim E_{n}$ is again an $\mathcal{O}_{X}$-sheaf, but the natural homomorphism $E \longrightarrow \lim E_{n}$ is in general not an isomorphism. However, if we restrict to coherent $\mathcal{O}_{X}$-modules $E$, then indeed $E \simeq \lim E_{n}$. This proves that a coherent $\mathcal{O}_{X}$-module is the same as an inverse system of coherent $\mathcal{O}_{X_{n}}$-sheaves $E_{n}$ together with transition maps $E_{n} \longrightarrow i_{m, n *} E_{m}$ inducing isomorphisms $i_{m, n}^{*} E_{n} \simeq E_{m}$ (see [6, II.9] or [11]).

By $\operatorname{Coh}(\mathcal{X}) \subset \mathcal{O}_{X}$-Mod we denote the full abelian subcategory of all coherent sheaves on $X$ and we tacitly use the equivalence of $\operatorname{Coh}(X)$ with the abelian category of coherent inverse systems as just explained. The restriction to $\mathcal{X}_{n}$ will be written as

$$
\operatorname{Coh}(X) \longrightarrow \operatorname{Coh}\left(X_{n}\right), \quad E \longmapsto E_{n} .
$$

So in particular, $E_{0} \in \mathbf{C o h}(X)$ will denote the restriction of a sheaf $E \in \operatorname{Coh}(X)$ or $E_{n} \in \operatorname{Coh}\left(X_{n}\right)$ to the special fibre $X=X_{0}$. As we assume our formal scheme to be smooth, any coherent sheaf on $\mathcal{X}$ admits locally a finite free resolution. However, since $\mathcal{X}$ is not necessarily projective, locally free resolutions might not exist globally.

The category $\operatorname{Coh}(\mathcal{X})$ of coherent sheaves on the formal $R$-scheme $X$ is in a natural way an $R$-linear category. A coherent sheaf $E \in \operatorname{Coh}(X)$ has support on $X_{n}$ if $t^{n+1} E=0$ and, as in the introduction, the subcategory consisting of all sheaves having support on some $\mathcal{X}_{n}$ is denoted by $\operatorname{Coh}(\mathcal{X})_{0}$.

A coherent sheaf $E \in \mathbf{C o h}(\mathcal{X})$ is $R$-flat if multiplication with $t$ yields an injective homomorphism $t: E \rightarrow E$. By $\operatorname{Coh}(\mathcal{X})_{\mathrm{f}} \subset \operatorname{Coh}(\mathcal{X})$ we denote the full additive subcategory of $R$-flat sheaves. This subcategory is clearly not abelian, but the two subcategories

$$
\operatorname{Coh}(X)_{0}, \operatorname{Coh}(X)_{\mathrm{f}} \subset \operatorname{Coh}(X)
$$

define a torsion theory for the abelian category $\operatorname{Coh}(\mathcal{X})$. More precisely, there are no non-trivial homomorphisms from objects in $\operatorname{Coh}(\mathcal{X})_{0}$ to objects in $\operatorname{Coh}(\mathcal{X})_{\mathrm{f}}$ and every $E \in \operatorname{Coh}(\mathcal{X})$ is in a unique way an extension

$$
0 \longrightarrow E_{\text {tor }} \longrightarrow E \longrightarrow E_{\mathrm{f}} \longrightarrow 0
$$

with $E_{\text {tor }} \in \operatorname{Coh}(X)_{0}$ and $E_{\mathrm{f}} \in \operatorname{Coh}(X)_{\mathrm{f}}$. Indeed, set $E_{\mathrm{tor}}:=\bigcup \operatorname{ker}\left(t^{n}: E \rightarrow E\right)$, i.e. the $R$-torsion subsheaf of $E$. The union must stabilize, as $E$ is coherent, and $E_{\mathrm{f}}:=E / E_{\text {tor }}$ is $R$-flat. (Note that in general this torsion theory is not cotilting, i.e. not every $R$-torsion sheaf is a quotient of an $R$-flat one.)

Let us now define the abelian category of coherent sheaves on the general fibre

$$
\operatorname{Coh}\left(\mathcal{X}_{K}\right):=\operatorname{Coh}(\mathcal{X}) / \operatorname{Coh}(X)_{0} .
$$


Remark 2.1. Since we divide out by a small subcategory, the quotient is a category with Homsets. The same remark applies to all later quotient constructions and we will henceforth ignore the issue.

The image of a sheaf $E \in \operatorname{Coh}(\mathcal{X})$ under the natural projection from $\operatorname{Coh}(\mathcal{X})$ onto $\operatorname{Coh}\left(\mathcal{X}_{K}\right)$ is denoted $E_{K}$.

For two coherent sheaves $E, E^{\prime} \in \operatorname{Coh}(\mathcal{X})$ we shall write $\operatorname{Hom}\left(E, E^{\prime}\right)$ for the group of homomorphisms in $\operatorname{Coh}(\mathcal{X})$ and $\operatorname{Hom}_{K}\left(E_{K}, E_{K}^{\prime}\right)$ for the group of homomorphisms of their images $E_{K}, E_{K}^{\prime}$ in $\operatorname{Coh}\left(\mathcal{X}_{K}\right)$. The natural homomorphisms induced by the projection will be denoted

$$
\eta: \operatorname{Hom}\left(E, E^{\prime}\right) \rightarrow \operatorname{Hom}_{K}\left(E_{K}, E_{K}^{\prime}\right)
$$

By construction of the quotient, any morphism $E_{K} \rightarrow E_{K}^{\prime}$ in $\operatorname{Coh}\left(\mathcal{X}_{K}\right)$ is an equivalence class of diagrams $\left(E \stackrel{s_{0}}{\longleftarrow} E_{0} \stackrel{g}{\longrightarrow} E^{\prime}\right)$ with $\operatorname{ker}\left(s_{0}\right), \operatorname{Coker}\left(s_{0}\right) \in \operatorname{Coh}(X)_{0}$. The composition

$$
\left(E \leftarrow E_{0} \longrightarrow E^{\prime}\right) \circ\left(E^{\prime} \longleftarrow E_{0}^{\prime} \longrightarrow E^{\prime \prime}\right)
$$

of two morphisms $E_{K} \longrightarrow E_{K}^{\prime}$ and $E_{K}^{\prime} \longrightarrow E_{K}^{\prime \prime}$ is naturally defined by means of the fibre product $\left(E \longleftarrow E_{0} \times E^{\prime} E_{0}^{\prime} \rightarrow E^{\prime \prime}\right)$.

Also note that $\operatorname{Coh}(\mathcal{X})_{\mathrm{f}} \rightarrow \operatorname{Coh}\left(\mathcal{X}_{K}\right)$ is essentially surjective, i.e. every object $F \in \operatorname{Coh}\left(\mathcal{X}_{K}\right)$ can be lifted to an $R$-flat sheaf on $\mathcal{X}$. Indeed, if $F=E_{K}$, then $\left(E_{\mathrm{f}}\right)_{K} \simeq E_{K}=F$ and, therefore, $E_{\mathrm{f}}$ is an $R$-flat lift of $F$.

Remark 2.2. As mentioned in the introduction, to the formal $R$-scheme $\mathcal{X}$ one can associate the general fibre $\mathcal{X}_{K}$ which is a rigid analytic space (see [2], [15], [16]). The abelian category $\operatorname{Coh}\left(\mathcal{X}_{K}\right)$ is in fact equivalent to the category of coherent sheaves on $X_{K}$, which explains the notation.

Proposition 2.3. The abelian category $\operatorname{Coh}\left(\mathcal{X}_{K}\right)$ is $K$-linear and for all $F, G \in$ $\operatorname{Coh}(\mathcal{X})$ the natural projection $\operatorname{Coh}(\mathcal{X}) \rightarrow \operatorname{Coh}\left(\mathcal{X}_{K}\right)$ induces a K-linear isomorphism

$$
\operatorname{Hom}(F, G) \otimes_{R} K \stackrel{\sim}{\longrightarrow} \operatorname{Hom}_{K}\left(F_{K}, G_{K}\right)
$$

Proof. As a quotient of the $R$-linear category $\operatorname{Coh}(\mathcal{X})$, the category $\operatorname{Coh}\left(\mathcal{X}_{K}\right)$ is also $R$-linear. The multiplication with $t^{-1}$ is defined as follows. Let $f \in$ $\operatorname{Hom}_{K}\left(F_{K}, G_{K}\right)$ be a morphism represented by $f:\left(F \stackrel{s_{0}}{\longleftarrow} F_{0} \stackrel{g}{\longrightarrow} G\right)$ with $\operatorname{Ker}\left(s_{0}\right), \operatorname{Coker}\left(s_{0}\right) \in \operatorname{Coh}(\mathcal{X})_{0}$. Then set $t^{-1} f:\left(F \stackrel{t s_{0}}{\longleftarrow} F_{0} \stackrel{g}{\longrightarrow} G\right)$, which is a well-defined morphism in $\operatorname{Coh}\left(\mathcal{X}_{K}\right)$. This is because the objects $\operatorname{Ker}\left(t s_{0}\right)$ and 
$\operatorname{Coker}\left(t s_{0}\right)$ are in $\operatorname{Coh}(\mathcal{X})_{0}$. Moreover, one has $t\left(t^{-1} f\right)=f$ due to the following commutative diagram

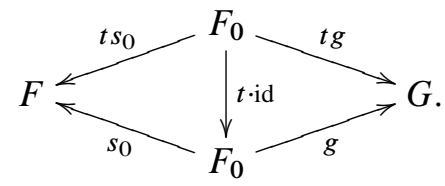

The $K$-linearity of the composition is obvious.

Consider now the induced $K$-linear map

$$
\eta_{K}: \operatorname{Hom}(F, G) \otimes_{R} K \longrightarrow \operatorname{Hom}_{K}\left(F_{K}, G_{K}\right)
$$

To prove the injectivity of $\eta_{K}$, let $f \in \operatorname{Hom}(F, G)$ with $\eta(f)=\eta_{K}(f)=0$. Then there exists a commutative diagram

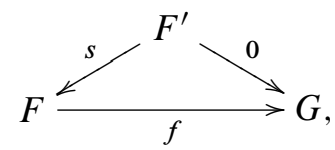

with $\operatorname{Ker}(s), \operatorname{Coker}(s) \in \operatorname{Coh}(\mathcal{X})_{0}$ and hence $f$ factorizes through

$$
f: F \stackrel{q}{\longrightarrow} \operatorname{Coker}(s) \stackrel{f^{\prime}}{\longrightarrow} G .
$$

Thus, if $t^{n} \operatorname{Coker}(s)=0$ for some $n>0$, then this yields $t^{n} f=f^{\prime} \circ\left(t^{n} q\right)=0$. In particular, $f \otimes 1 \in \operatorname{Hom}(F, G) \otimes K$ is trivial.

In order to prove the surjectivity of $\eta_{K}$, we have to show that for any $f \in$ $\operatorname{Hom}_{K}\left(F_{K}, G_{K}\right)$ there exists an integer $k$, such that $t^{k} f$ is induced by a morphism $F \longrightarrow G$ in $\operatorname{Coh}(X)$. Write $f:\left(F<\stackrel{s_{0}}{<} F_{0} \stackrel{g}{\longrightarrow} G\right)$ with $t^{n} \operatorname{Ker}\left(s_{0}\right)=$ $t^{m} \operatorname{Coker}\left(s_{0}\right)=0$ for some positive integers $m, n$. Consider the exact sequence

$$
0 \longrightarrow \operatorname{Hom}\left(F^{\prime}, G\right) \stackrel{\circ p}{\longrightarrow} \operatorname{Hom}\left(F_{0}, G\right) \stackrel{\circ i}{\longrightarrow} \operatorname{Hom}\left(\operatorname{Ker}\left(s_{0}\right), G\right)
$$

which is induced by the natural projection $p: F_{0} \longrightarrow F^{\prime}:=\operatorname{im}\left(s_{0}\right)$ and by its kernel $i: \operatorname{ker}\left(s_{0}\right) \hookrightarrow F_{0}$. Since $\left(t^{n} g\right) \circ i=g \circ\left(t^{n} i\right)=0$, there exists a (unique) homomorphism $g^{\prime}: F^{\prime} \longrightarrow G$ such that $g^{\prime} \circ p=t^{n} g$. This yields the commutative diagram

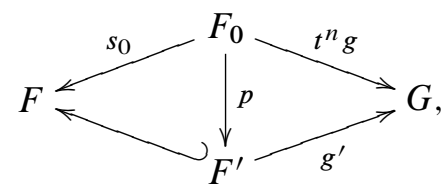


which allows one to represent $t^{n} f$ by $\left(F \ll F^{\prime} \stackrel{g^{\prime}}{\longrightarrow} G\right)$.

As $F / F^{\prime} \simeq \operatorname{Coker}\left(s_{0}\right)$ is annihilated by $t^{m}$, the homomorphism $t^{m} g^{\prime}: F^{\prime} \longrightarrow G$ lifts to a homomorphism $g^{\prime \prime}: F \longrightarrow G$, i.e. $\left.g^{\prime \prime}\right|_{F^{\prime}}=t^{m} g^{\prime}$. This yields the commutative diagram

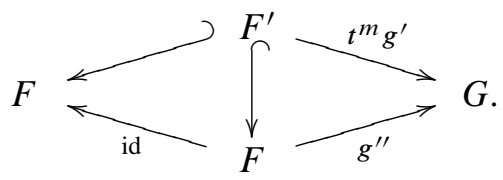

Hence $t^{m+n} f$ is represented by $\left(F \stackrel{\text { id }}{\longleftarrow} F \stackrel{g^{\prime \prime}}{\longrightarrow} G\right)$, i.e. $t^{m+n} f=\eta\left(g^{\prime \prime}\right)$.

2.2. The derived category of the general fibre. Let $\pi: \mathcal{X} \rightarrow \operatorname{Spf}(R)$ be a formal deformation of $X$ and consider the bounded derived category of $\mathcal{X}$ defined as

$$
\mathrm{D}^{\mathrm{b}}(\mathcal{X}):=\mathrm{D}_{\mathrm{coh}}^{\mathrm{b}}\left(\mathcal{O}_{X}-\mathbf{M o d}\right)
$$

which by definition is an $R$-linear triangulated category.

Remark 2.4. We will always tacitly use the well-known (at least for schemes) fact that any bounded complex with coherent cohomology on a smooth formal scheme is perfect, i.e. locally isomorphic to a finite complex of locally free sheaves of finite type (see e.g. [10, Corollary 5.9]). In other words $\mathrm{D}_{\text {perf }}(\mathcal{X}) \simeq \mathrm{D}^{\mathrm{b}}(\mathcal{X})$. This is however not true for $X_{n}, n>0$. Indeed, e.g. for $n=1$ one has $\operatorname{Tor}_{R_{1}}^{i}\left(R_{0}, R_{0}\right) \simeq R_{0}$ for all $i \geq 0$. So, the $R_{1}$-module $R_{0}$ does not admit a finite free resolution. So we will have to work with

$$
\mathrm{D}_{\text {perf }}\left(X_{n}\right) \subset \mathrm{D}^{\mathrm{b}}\left(X_{n}\right)
$$

the full triangulated subcategory of perfect complexes on $X_{n}$.

Recall that for the noetherian scheme $\mathcal{X}_{n}$ the functor

$$
\mathrm{D}^{\mathrm{b}}\left(\operatorname{Coh}\left(\mathcal{X}_{n}\right)\right) \stackrel{\sim}{\sim} \mathrm{D}^{\mathrm{b}}\left(\mathcal{X}_{n}\right):=\mathrm{D}_{\mathrm{coh}}^{\mathrm{b}}\left(\mathcal{O}_{X_{n}}-\mathbf{M o d}\right)
$$

is an equivalence. Contrary to the case of a noetherian scheme, the natural functor

$$
\mathrm{D}^{\mathrm{b}}(\operatorname{Coh}(\mathcal{X})) \longrightarrow \mathrm{D}^{\mathrm{b}}(\mathcal{X})=\mathrm{D}_{\text {coh }}^{\mathrm{b}}\left(\mathcal{O}_{X}-\mathbf{M o d}\right)
$$

is in general not an equivalence. However, (2.1) induces an equivalence between the full subcategories of $R$-torsion complexes. To be more precise, let

$$
\mathrm{D}_{0}^{\mathrm{b}}(\mathcal{X}):=\mathrm{D}_{\mathbf{C o h}(\mathcal{x})_{0}}^{\mathrm{b}}\left(\mathcal{O}_{X}-\mathbf{M o d}\right) \subset \mathrm{D}^{\mathrm{b}}(\mathcal{X}) \quad \text { and } \quad \mathrm{D}_{0}^{\mathrm{b}}(\operatorname{Coh}(\mathcal{X})) \subset \mathrm{D}^{\mathrm{b}}(\boldsymbol{C o h}(\mathcal{X}))
$$

be the full triangulated subcategories of complexes with cohomology contained in $\operatorname{Coh}(\mathcal{X})_{0}$. Then one has: 
Proposition 2.5. i) The natural functor $\mathrm{D}^{\mathrm{b}}\left(\operatorname{Coh}(\mathcal{X})_{0}\right) \rightarrow \mathrm{D}^{\mathrm{b}}(\operatorname{Coh}(\mathcal{X}))$ induces an equivalence

$$
\mathrm{D}^{\mathrm{b}}\left(\operatorname{Coh}(X)_{0}\right) \stackrel{\sim}{\longrightarrow} \mathrm{D}_{0}^{\mathrm{b}}(\operatorname{Coh}(X)) .
$$

ii) The natural functor $\mathrm{D}^{\mathrm{b}}\left(\mathbf{C o h}(\mathcal{X})_{0}\right) \rightarrow \mathrm{D}^{\mathrm{b}}(\mathcal{X})$ induces an equivalence

$$
\mathrm{D}^{\mathrm{b}}\left(\operatorname{Coh}(X)_{0}\right) \stackrel{\sim}{\longrightarrow} \mathrm{D}_{0}^{\mathrm{b}}(X) .
$$

Proof. i) It suffices to show (see the dual version of [7, Lemma 3.6]) that for any monomorphism $f: E \hookrightarrow E^{\prime}$ in $\operatorname{Coh}(\mathcal{X})$, with $E \in \operatorname{Coh}(\mathcal{X})_{0}$, there exists $g: E^{\prime} \longrightarrow E^{\prime \prime}$, with $E^{\prime \prime} \in \mathbf{C o h}(\mathcal{X})_{0}$ such that $g \circ f$ is injective.

By the Artin-Rees Lemma, we know that the filtration $E_{k}:=E \cap t^{k} E^{\prime}$ is $t$-stable, that is, there is some $n \in \mathbb{N}$ such that $t E_{k}=E_{k+1}$, whenever $k \geq n$. Let $\ell$ be a positive integer such that $t^{\ell} E=0$ and let $g: E^{\prime} \longrightarrow E^{\prime \prime}:=E^{\prime} / t^{n+\ell} E^{\prime}$ be the projection. The composition $g \circ f$ is injective, as $\operatorname{ker}(g \circ f)=E_{n+\ell}=$ $t^{\ell} E_{n} \hookrightarrow t^{\ell} E=0$.

ii) We follow Yekutieli [19], but see also [1]. Let $\mathbf{Q C o h}(\mathcal{X}) \subset \mathcal{O}_{X}$-Mod be the full abelian subcategory of quasi-coherent sheaves on $\mathcal{X}$, i.e. of sheaves which are locally cokernels of $\mathcal{O}_{X}^{I} \rightarrow \mathcal{O}_{X}^{J}$ for some index sets $I, J$. Then define $\mathbf{Q C o h}(X)_{\mathrm{d}} \subset$ $\operatorname{QCoh}(X)$ as the full thick abelian subcategory of discrete quasi-coherent sheaves (see the Appendix for the definition of thick abelian subcategory). By definition, a sheaf $E$ on $\mathcal{X}$ is discrete if the natural functor $\Gamma_{\mathrm{d}}(E):=\lim \mathscr{H o m}\left(\mathcal{O}_{X_{n}}, E\right) \rightarrow E$ is an isomorphism.

Clearly, a coherent sheaf on $\mathcal{X}$ is discrete if and only if it is $R$-torsion, i.e. $\operatorname{Coh}(\mathcal{X})_{0}=\operatorname{Coh}(\mathcal{X}) \cap \operatorname{QCoh}(\mathcal{X})_{\mathrm{d}}$ which is a thick subcategory of $\mathbf{Q C o h}(\mathcal{X})_{\mathrm{d}}$. Moreover, by [19, Proposition 3.8] every $E \in \mathbf{Q C o h}(X)_{\mathrm{d}}$ is the limit of coherent $R$-torsion sheaves. Thus $\mathrm{D}_{\operatorname{Coh}(X)_{0}}^{\mathrm{b}}\left(\mathbf{Q C o h}(X)_{\mathrm{d}}\right)$ is the same as $\mathrm{D}_{\text {coh }}^{\mathrm{b}}\left(\mathbf{Q C o h}(X)_{\mathrm{d}}\right)$. Lemma 2.6 below gives an equivalence $\mathrm{D}^{\mathrm{b}}\left(\mathbf{C o h}(X)_{0}\right) \simeq \mathrm{D}_{\mathbf{C o h}(X)_{0}}^{\mathrm{b}}\left(\mathbf{Q C o h}(X)_{\mathrm{d}}\right)$. Hence we conclude the equivalence $\mathrm{D}^{\mathrm{b}}\left(\mathbf{C o h}(\mathcal{X})_{0}\right) \simeq \mathrm{D}_{\text {coh }}^{\mathrm{b}}\left(\mathbf{Q} \operatorname{Coh}(X)_{\mathrm{d}}\right)$.

Finally, one applies [19, Theorem 4.8] which asserts that the natural functor induces an equivalence of $\mathrm{D}^{\mathrm{b}}\left(\mathbf{Q} \operatorname{Coh}(X)_{\mathrm{d}}\right)$ with the full triangulated subcategory of $\mathrm{D}^{\mathrm{b}}\left(\mathcal{O}_{X}\right.$-Mod) of all complexes with cohomology in $\mathbf{Q C o h}(\mathcal{X})_{\mathrm{d}}$. (The inverse functor is given by $R \Gamma_{\mathrm{d}}$.) Adding the condition that the cohomology be coherent proves ii).

Lemma 2.6. Let $\mathcal{A} \subseteq \mathscr{B}$ be a full thick abelian subcategory of an abelian category $\mathscr{B}$ with infinite direct sums. Assume that every object of $\mathcal{B}$ is the direct limit of its subobjects belonging to $\mathcal{A}$ and that $\mathcal{A}$ is noetherian (i.e. every ascending sequence of subobjects is stationary). Then the natural functor yields an equivalence

$$
\mathrm{D}^{\mathrm{b}}(\mathcal{A}) \stackrel{\sim}{\longrightarrow} \mathrm{D}_{\mathcal{A}}^{\mathrm{b}}(\mathscr{B}),
$$


where $\mathrm{D}_{\mathscr{A}}^{\mathrm{b}}(\mathscr{B})$ is the full triangulated subcategory of $\mathrm{D}^{\mathrm{b}}(\mathscr{B})$ of complexes with cohomology in $\mathcal{A}$.

Proof. Let $f: E \longrightarrow E^{\prime}$ be a surjection in $\mathscr{B}$, with $E^{\prime} \in \mathcal{A}$. We need to show that there exists a morphism $g: G \longrightarrow E$ with $G \in \mathcal{A}$ such that $f \circ g: G \longrightarrow E^{\prime}$ is again surjective (see e.g. [7, Lemma 3.6]).

By assumption, there exists a direct system of objects $\left\{E_{i}\right\}$ in $\mathcal{A}$ such that $\lim E_{i} \simeq$ $E$. Hence, there exists a surjection $j: \bigoplus_{i} E_{i} \longrightarrow E \longrightarrow E^{\prime}$.

Then let $E_{k}^{\prime}:=\operatorname{im}\left(\bigoplus_{i=0}^{k} E_{i} \longrightarrow E \longrightarrow E^{\prime}\right)$, which form an ascending sequence of subobjects of $E^{\prime}$. Since $\mathcal{A}$ is a noetherian, the sequence $\left\{E_{k}^{\prime}\right\}$ stabilizes, and, as $j$ is surjective, $E_{k}^{\prime}=E^{\prime}$ for $k \gg 0$. Then set $G:=\bigoplus_{i=0}^{k} E_{i} \in \mathcal{A}$, for some $k \gg 0$, and let $g$ be the natural morphism.

Remark 2.7. i) The equivalences of Proposition 2.5 put in one diagram read

$$
\mathrm{D}^{\mathrm{b}}\left(\operatorname{Coh}(X)_{0}\right) \simeq \mathrm{D}_{0}^{\mathrm{b}}(\operatorname{Coh}(\mathcal{X})) \simeq \mathrm{D}_{0}^{\mathrm{b}}(\mathcal{X}) .
$$

ii) The categories $\mathrm{D}_{0}^{\mathrm{b}}(\mathcal{X}) \subset \mathrm{D}^{\mathrm{b}}(\mathcal{X})$ and $\mathrm{D}^{\mathrm{b}}\left(\boldsymbol{C o h}(\mathcal{X})_{0}\right) \subset \mathrm{D}^{\mathrm{b}}(\boldsymbol{C o h}(\mathcal{X}))$ can also be described as the smallest full triangulated subcategories containing all $R$-torsion coherent sheaves. Here, a sheaf $E \in \operatorname{Coh}\left(\mathcal{X}_{n}\right)$ is at the same time considered as an object in $\mathrm{D}^{\mathrm{b}}(\mathcal{X})$ and $\mathrm{D}^{\mathrm{b}}(\mathbf{C o h}(\mathcal{X}))$. This is clear, as any bounded complex with $R$-torsion cohomology can be filtered (in the triangulated sense) with quotients being translates of such sheaves.

In the introduction we have already defined the derived category of the general fibre $\mathrm{D}^{\mathrm{b}}\left(\mathcal{X}_{K}\right)$, i.e. the Verdier quotient

$$
\mathrm{D}^{\mathrm{b}}\left(\mathcal{X}_{K}\right):=\mathrm{D}^{\mathrm{b}}(\mathcal{X}) / \mathrm{D}_{0}^{\mathrm{b}}(\mathcal{X})=\mathrm{D}_{\text {coh }}^{\mathrm{b}}\left(\mathcal{O}_{X}-\mathbf{M o d}\right) / \mathrm{D}_{\mathbf{C o h}(X)_{0}}^{\mathrm{b}}\left(\mathcal{O}_{X}-\mathbf{M o d}\right) .
$$

One can also consider the quotient $\mathrm{D}^{\mathrm{b}}(\mathbf{C o h}(\mathcal{X})) / \mathrm{D}_{0}^{\mathrm{b}}(\mathbf{C o h}(X))$ which, for a lack of a better notation, will be called

$$
\mathrm{D}^{\mathrm{b}}\left(\mathcal{X}_{K}^{c}\right):=\mathrm{D}^{\mathrm{b}}(\operatorname{Coh}(\mathcal{X})) / \mathrm{D}_{0}^{\mathrm{b}}(\operatorname{Coh}(\mathcal{X})) .
$$

(For a thorough discussion of the Verdier quotient see the Appendix.)

In both cases, the quotients are triangulated and the natural projections

$$
\mathrm{D}^{\mathrm{b}}(\mathcal{X}) \longrightarrow \mathrm{D}^{\mathrm{b}}\left(\mathcal{X}_{K}\right) \text { and } \mathrm{D}^{\mathrm{b}}(\mathbf{C o h}(\mathcal{X})) \longrightarrow \mathrm{D}^{\mathrm{b}}\left(\mathcal{X}_{K}^{c}\right)
$$

are exact. The image of a complex $E$ under any of these projections shall be denoted $E_{K}$. 
Remark 2.8. As $\operatorname{Coh}(\mathcal{X})_{0} \subset \operatorname{Coh}(\mathcal{X}) \subset \mathcal{O}_{X}$-Mod are Serre subcategories, the subcategories $\mathrm{D}_{0}^{\mathrm{b}}\left(\mathcal{O}_{X}\right.$-Mod $) \subset \mathrm{D}^{\mathrm{b}}(\mathcal{X})$ and $\mathrm{D}_{0}^{\mathrm{b}}(\operatorname{Coh}(\mathcal{X})) \subset \mathrm{D}^{\mathrm{b}}(\operatorname{Coh}(\mathcal{X}))$ are thick. This means that the direct summands of their objects are again contained in the subcategories. This has the consequence that the kernel of the two projections in (2.3) are indeed $\mathrm{D}_{0}^{\mathrm{b}}(\mathcal{X})$ and $\mathrm{D}_{0}^{\mathrm{b}}(\boldsymbol{C o h}(\mathcal{X}))$ respectively.

Proposition 2.9. The triangulated category $\mathrm{D}^{\mathrm{b}}\left(\mathcal{X}_{K}\right)$ is $K$-linear and for all $E, E^{\prime} \in$ $\mathrm{D}^{\mathrm{b}}(\mathcal{X})$ the natural projection $\mathrm{D}^{\mathrm{b}}(\mathcal{X}) \rightarrow \mathrm{D}^{\mathrm{b}}\left(\mathcal{X}_{K}\right)$ induces $K$-linear isomorphisms

$$
\operatorname{Hom}_{D^{\mathrm{b}}(\mathcal{X})}\left(E, E^{\prime}\right) \otimes_{R} K \stackrel{\sim}{\rightarrow} \operatorname{Hom}_{\mathrm{D}^{\mathrm{b}}\left(\mathcal{X}_{K}\right)}\left(E_{K}, E_{K}^{\prime}\right) .
$$

Similarly, $\mathrm{D}^{\mathrm{b}}\left(\mathcal{X}_{K}^{c}\right)$ is $K$-linear and for $E, E^{\prime} \in \mathrm{D}^{\mathrm{b}}(\mathbf{C o h}(\mathcal{X}))$ one has

$$
\operatorname{Hom}_{\mathrm{D}^{\mathrm{b}}(\operatorname{Coh}(X))}\left(E, E^{\prime}\right) \otimes_{R} K \stackrel{\sim}{\rightarrow} \operatorname{Hom}_{\mathrm{D}^{\mathrm{b}}\left(X_{K}^{c}\right)}\left(E_{K}, E_{K}^{\prime}\right) .
$$

In particular, $\mathrm{D}^{\mathrm{b}}\left(\mathcal{X}_{K}\right)$ and $\mathrm{D}^{\mathrm{b}}\left(\mathcal{X}_{K}^{c}\right)$ have finite-dimensional Hom-spaces over $K$.

Proof. As we work with bounded complexes, the proof of Proposition 2.3 carries over.

2.3. Derived functors and Fourier-Mukai transforms. First of all we prove that the usual derived functors (tensor product, pull-back push-forward, Hom's) are welldefined in the geometric setting we are dealing with.

Proposition 2.10. Let $f, g: \mathcal{X} \rightarrow \mathcal{X}^{\prime}$ be morphisms of smooth and proper formal schemes over $\operatorname{Spf}(R)$ and assume $f$ to be proper. Then the following R-linear functors are defined:

$$
\begin{aligned}
& R \mathscr{H o m}_{X}(-,-): \mathrm{D}^{\mathrm{b}}(\mathcal{X})^{\mathrm{op}} \times \mathrm{D}^{\mathrm{b}}(\mathcal{X}) \longrightarrow \mathrm{D}^{\mathrm{b}}(\mathcal{X}), \\
&(-) \otimes{ }^{L}(-): \mathrm{D}^{\mathrm{b}}(\mathcal{X}) \times \mathrm{D}^{\mathrm{b}}(\mathcal{X}) \longrightarrow \mathrm{D}^{\mathrm{b}}(\mathcal{X}), \\
& L g^{*}: \mathrm{D}^{\mathrm{b}}\left(\mathcal{X}^{\prime}\right) \longrightarrow \mathrm{D}^{\mathrm{b}}(\mathcal{X}), \\
& R f_{*}: \mathrm{D}^{\mathrm{b}}(\mathcal{X}) \longrightarrow \mathrm{D}^{\mathrm{b}}\left(X^{\prime}\right), \\
& R \operatorname{Hom}_{\mathrm{D}^{\mathrm{b}}(\mathcal{X})}(-,-): \mathrm{D}^{\mathrm{b}}(\mathcal{X})^{\mathrm{op}} \times \mathrm{D}^{\mathrm{b}}(\mathcal{X}) \longrightarrow \mathrm{D}^{\mathrm{b}}(R-\bmod ),
\end{aligned}
$$

where we denote by $R$-mod the abelian category of $R$-modules of finite rank.

Proof. Due to [17], the functors previously considered are all well-defined if we work with unbounded derived categories of modules $\mathrm{D}\left(\mathcal{O}_{x}\right.$-Mod $), \mathrm{D}\left(\mathcal{O}_{X^{\prime}}\right.$-Mod $)$ and $\mathrm{D}(R$-Mod) (here $R$-Mod denotes the abelian category of $R$-modules). To prove the proposition, we only have to show that, by restricting the domain to the corresponding derived categories of bounded complexes with coherent cohomology, the images of these functors are still the bounded derived categories of complexes with coherent cohomology. This is clear since all complexes are perfect. 
All the basic properties of the functors considered in the previous proposition (e.g. commutativity, flat base change, projection formula) hold in the formal context. For an object $E \in \mathrm{D}^{\mathrm{b}}(\mathcal{X})$ a trace map $\operatorname{tr}_{E}: E^{\searrow} \otimes E \rightarrow \mathcal{O}_{X}$ is well-defined (see [10]).

Passing to the triangulated category $\mathrm{D}^{\mathrm{b}}\left(\mathcal{X}_{K}\right)$ of the generic fibre, the result in Proposition 2.10 still hold. Indeed, all the functors are $R$-linear and hence they factorize through the category $\mathrm{D}^{\mathrm{b}}\left(\mathcal{X}_{K}\right)$. Indeed, $\mathcal{F} \in \mathrm{D}^{\mathrm{b}}(\mathcal{X})$ is contained in $\mathrm{D}_{0}^{\mathrm{b}}(\mathcal{X})$ if and only if $t^{n} \mathcal{F}=0$ for $n \gg 0$. Since the functors are $R$-linear, the same would hold for the image of $\mathcal{F}$ which would therefore as well be contained in the subcategory $\mathrm{D}_{0}^{\mathrm{b}}$. Thus we get the following list of functors:

$$
\begin{aligned}
R \operatorname{Hom}_{X_{K}}(-,-) & : \mathrm{D}^{\mathrm{b}}\left(\mathcal{X}_{K}\right)^{\mathrm{op}} \times \mathrm{D}^{\mathrm{b}}\left(\mathcal{X}_{K}\right) \longrightarrow \mathrm{D}^{\mathrm{b}}\left(\mathcal{X}_{K}\right), \\
(-) \otimes^{L}(-): & \mathrm{D}^{\mathrm{b}}\left(\mathcal{X}_{K}\right) \times \mathrm{D}^{\mathrm{b}}\left(\mathcal{X}_{K}\right) \longrightarrow \mathrm{D}^{\mathrm{b}}\left(\mathcal{X}_{K}\right), \\
L g^{*}: & \mathrm{D}^{\mathrm{b}}\left(\mathcal{X}_{K}^{\prime}\right) \longrightarrow \mathrm{D}^{\mathrm{b}}\left(\mathcal{X}_{K}\right), \\
R f_{*}: & \mathrm{D}^{\mathrm{b}}\left(\mathcal{X}_{K}\right) \longrightarrow \mathrm{D}^{\mathrm{b}}\left(\mathcal{X}_{K}^{\prime}\right), \\
R \operatorname{Hom}_{\mathrm{D}^{\mathrm{b}}\left(\mathcal{X}_{K}\right)}(-,-): & \mathrm{D}^{\mathrm{b}}\left(\mathcal{X}_{K}\right)^{\mathrm{op}} \times \mathrm{D}^{\mathrm{b}}\left(\mathcal{X}_{K}\right) \longrightarrow \mathrm{D}^{\mathrm{b}}(K-\text { vect }),
\end{aligned}
$$

where we denote by $K$-vect the abelian category of finite dimensional $K$-vector spaces. Of course, all the usual relations between these functors continue to hold in $\mathrm{D}^{\mathrm{b}}\left(\mathcal{X}_{K}\right)$. In particular, given an object $E_{K} \in \mathrm{D}^{\mathrm{b}}\left(\mathcal{X}_{K}\right)$, its $d u a l E_{K}^{\vee} \in \mathrm{D}^{\mathrm{b}}\left(\mathcal{X}_{K}\right)$ is welldefined and $E_{K}^{\sim} \simeq E_{K}$. Moreover, we have a trace map $\operatorname{tr}_{E_{K}}: E_{K}^{\vee} \otimes E_{K} \longrightarrow \mathcal{O}_{\mathcal{X}_{K}}$, where $\mathcal{O}_{X_{K}}$ is the image of $\mathcal{O}_{X}$ in $\mathrm{D}^{\mathrm{b}}\left(\mathcal{X}_{K}\right)$.

Using those facts, we define Fourier-Mukai functors for formal deformations or for the derived categories of the general fibres. Indeed, consider two smooth and proper formal schemes $\mathcal{X} \rightarrow \operatorname{Spf}(R)$ and $\mathcal{X}^{\prime} \rightarrow \operatorname{Spf}(R)$ of dimension $d$ respectively $d^{\prime}$, with special fibres $X$ respectively $X^{\prime}$. The fibre product $\mathcal{X} \times_{R} \mathcal{X}^{\prime} \rightarrow \operatorname{Spf}(R)$, described by the inductive system $\mathcal{X}_{n} \times{ }_{R_{n}} \mathcal{X}_{n}^{\prime}$, is again smooth and proper and its special fibre is $X \times X^{\prime}$. The two projections shall be called $q: \mathcal{X} \times_{R} X^{\prime} \longrightarrow X$ and $p: \mathcal{X} \times_{R} X^{\prime} \rightarrow \mathcal{X}^{\prime}$.

Let $\mathscr{E} \in \mathrm{D}^{\mathrm{b}}\left(\mathcal{X} \times_{R} \mathcal{X}^{\prime}\right)$. Due to the results in the previous section, one can consider the induced Fourier-Mukai transform

$$
\Phi_{\mathcal{E}}: \mathrm{D}^{\mathrm{b}}(\mathcal{X}) \longrightarrow \mathrm{D}^{\mathrm{b}}\left(\mathcal{X}^{\prime}\right), \quad E \longmapsto R p_{*}\left(q^{*} E \otimes^{L} \mathcal{E}\right)
$$

As before, $\Phi_{\varepsilon}$ is $R$-linear, for $\mathcal{E}$ lives on the fibre product over $\operatorname{Spf}(R)$.

Now, for two given Fourier-Mukai transforms $\Phi_{\varepsilon}: \mathrm{D}^{\mathrm{b}}(\mathcal{X}) \rightarrow \mathrm{D}^{\mathrm{b}}\left(\mathcal{X}^{\prime}\right)$ and $\Phi_{\mathscr{F}}: \mathrm{D}^{\mathrm{b}}\left(\mathcal{X}^{\prime}\right) \longrightarrow \mathrm{D}^{\mathrm{b}}\left(\mathcal{X}^{\prime \prime}\right)$ with $\mathcal{X}^{\prime \prime}$ a smooth and proper formal scheme over $\operatorname{Spf}(R)$, the composition $\Phi_{\mathscr{F}} \circ \Phi_{\mathcal{E}}$ is again a Fourier-Mukai transform with kernel $\mathcal{F} * \mathcal{E}:=$ $\left(p_{\mathcal{X}, X^{\prime \prime}}\right)_{*}(\mathscr{\nabla} \mathcal{F})$, where $p_{\mathcal{X}, X^{\prime \prime}}: \mathcal{X} \times \mathcal{X}^{\prime} \times \mathcal{X}^{\prime \prime} \longrightarrow \mathcal{X} \times \mathcal{X}^{\prime \prime}$ is the natural projection.

Left and right adjoint functors of a Fourier-Mukai transform $\Phi_{\mathcal{E}}$ can be constructed as Fourier-Mukai transforms as follows. The left adjoint $\Phi_{\mathcal{E}_{\mathrm{L}}}$ and the right 
adjoint $\Phi_{\varepsilon_{\mathrm{R}}}$ are the Fourier-Mukai transforms with kernel

$$
\mathcal{E}_{\mathrm{L}}:=\mathcal{E}^{\vee} \otimes p^{*} \omega_{p}\left[d^{\prime}\right] \quad \text { respectively } \quad \mathcal{E}_{\mathrm{R}}:=\mathcal{E}^{\vee} \otimes q^{*} \omega_{q}[d],
$$

where $d=\operatorname{dim}(\mathcal{X})$ and $d^{\prime}=\operatorname{dim}\left(\mathcal{X}^{\prime}\right)$. (To adapt to this context the standard proof that those kernels define the left and right adjoints of $\Phi_{\mathcal{E}}$, we actually need Lemma 3.4 which will be proved later.)

The adjunction morphisms $\Phi_{\mathcal{E}_{\mathrm{L}}} \circ \Phi_{\mathcal{E}} \longrightarrow \mathrm{id}_{\mathrm{D}^{\mathrm{b}}(\mathcal{X})}$ and $\Phi_{\mathcal{E}} \circ \Phi_{\mathcal{E}_{\mathrm{R}}} \rightarrow \mathrm{id}_{\mathrm{D}^{\mathrm{b}}\left(\mathcal{X}^{\prime}\right)}$ are isomorphisms if and only if the natural morphisms $\operatorname{tr} x: \mathcal{E}_{\mathrm{L}} * \mathcal{E} \rightarrow \mathcal{O}_{\Delta_{X}}$ respectively $\operatorname{tr} x^{\prime}: E^{*} * \mathcal{E}_{\mathrm{R}} \rightarrow \mathcal{O}_{\Delta_{X}}$ induced by the trace morphisms are isomorphisms. Here $\Delta x$ and $\Delta X^{\prime}$ denote the relative diagonals in $\mathcal{X} \times_{R} \mathcal{X}$ respectively $\mathcal{X}^{\prime} \times_{R} \mathcal{X}^{\prime}$. (Sometimes (see e.g. [5]) the construction of the adjunction morphisms uses Grothendieck-Verdier duality for certain embeddings, e.g. for $\mathcal{X} \times_{R} \mathcal{X}^{\prime} \longleftrightarrow \mathcal{X} \times_{R} \mathcal{X}^{\prime} \times_{R} \mathcal{X}$. This can easily be replaced by an argument using relative duality over $R$ in the sense of Lemma 3.4 for the two sides. We leave the details to the reader.)

Remark 2.11. Everything said above is also valid for the non-reduced schemes $X_{n} \rightarrow \operatorname{Spec}\left(R_{n}\right)$ and $X_{n}^{\prime} \rightarrow \operatorname{Spec}\left(R_{n}\right)$ with the only difference that we have to assume now that the Fourier-Mukai kernel $\varepsilon_{n} \in \mathrm{D}^{\mathrm{b}}\left(\mathcal{X}_{n} \times{ }_{R_{n}} \mathcal{X}_{n}^{\prime}\right)$ is perfect. Then one can consider the two $R_{n}$-linear functors

$$
\Phi_{\mathcal{E}_{n}}: \mathrm{D}^{\mathrm{b}}\left(\mathcal{X}_{n}\right) \rightarrow \mathrm{D}^{\mathrm{b}}\left(\mathcal{X}_{n}^{\prime}\right) \text { and } \Phi_{\mathcal{E}_{n}}: \mathrm{D}_{\text {perf }}\left(\mathcal{X}_{n}\right) \rightarrow \mathrm{D}_{\text {perf }}\left(\mathcal{X}_{n}^{\prime}\right)
$$

Analogously, one wants to define the Fourier-Mukai transform

$$
\Phi_{\mathscr{F}}: \mathrm{D}^{\mathrm{b}}\left(\mathcal{X}_{K}\right) \longrightarrow \mathrm{D}^{\mathrm{b}}\left(\mathcal{X}_{K}^{\prime}\right)
$$

associated to an object $\mathcal{F} \in \mathrm{D}^{\mathrm{b}}\left(\left(\mathcal{X} \times_{R} \mathcal{X}^{\prime}\right)_{K}\right)$.

As the objects of $\mathrm{D}^{\mathrm{b}}\left(\mathcal{X} \times_{R} \mathcal{X}^{\prime}\right)$ are the same as those of $\mathrm{D}^{\mathrm{b}}\left(\left(\mathcal{X} \times_{R} \mathcal{X}^{\prime}\right)_{K}\right)$ (see the Appendix $)$, take $\mathscr{E} \in \mathrm{D}^{\mathrm{b}}\left(\mathcal{X} \times_{R} \mathcal{X}^{\prime}\right)$ such that $\mathcal{E}_{K} \simeq \mathcal{F}$. Then, by the $R$-linearity, the Fourier-Mukai transform $\Phi_{\mathcal{E}}: \mathrm{D}^{\mathrm{b}}(\mathcal{X}) \rightarrow \mathrm{D}^{\mathrm{b}}\left(\mathcal{X}^{\prime}\right)$ descends to a Fourier-Mukai transform $\Phi_{\mathcal{F}}: \mathrm{D}^{\mathrm{b}}\left(\mathcal{X}_{K}\right) \rightarrow \mathrm{D}^{\mathrm{b}}\left(\mathcal{X}_{K}^{\prime}\right)$, i.e. one has a commutative diagram

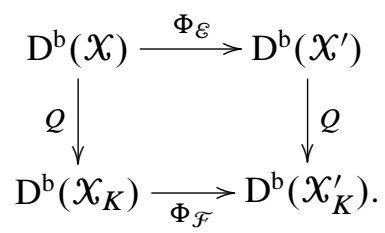

Since the objects of $\mathrm{D}^{\mathrm{b}}(\mathcal{X})$ and $\mathrm{D}^{\mathrm{b}}\left(\mathcal{X}_{K}\right)$ coincide, it is enough to check that $\Phi_{\mathcal{E}}\left(\mathrm{D}_{0}^{\mathrm{b}}(\mathcal{X})\right)=\mathrm{D}_{0}^{\mathrm{b}}\left(\mathcal{X}^{\prime}\right)$. Indeed, $\mathscr{G} \in \mathrm{D}^{\mathrm{b}}(\mathcal{X})$ is contained in $\mathrm{D}_{0}^{\mathrm{b}}(\mathcal{X})$ if and only if $t^{n} \mathscr{E}=0$ for $n \gg 0$. As $\Phi_{\mathscr{E}}$ is $R$-linear, this would imply $t^{n} \Phi_{\mathscr{E}}(\mathscr{E})=0$ and hence 
$\Phi_{\mathcal{E}}(\mathscr{E}) \in \mathrm{D}_{0}^{\mathrm{b}}\left(X^{\prime}\right)$. The behavior of $\Phi_{\mathcal{F}}$ on the level of morphisms is determined by Proposition 2.9. Moreover, $\Phi_{\mathcal{F}}$ is independent of the chosen lift $\mathcal{E}$. From this, it follows that right and left adjoints of Fourier-Mukai transforms as well as trace maps pass to the triangulated category of the generic fibre.

2.4. Fourier-Mukai equivalences of the general fibre. Here we will show that if the kernel of a Fourier-Mukai equivalence deforms to a complex on some finite order deformation or even to the general fibre, then it still induces derived equivalences of the finite order deformations or general fibres, respectively. This is certainly expected, as 'being an equivalence' should be an open property and indeed the proof follows the standard arguments.

Consider two smooth and proper formal schemes $\mathcal{X} \rightarrow \operatorname{Spf}(R)$ and $\mathcal{X}^{\prime} \rightarrow \operatorname{Spf}(R)$, with special fibres $X$ respectively $X^{\prime}$. The fibre product $X \times_{R} X^{\prime} \rightarrow \operatorname{Spf}(R)$, described by the inductive system $\mathcal{X}_{n} \times_{R_{n}} X_{n}^{\prime} \rightarrow \operatorname{Spec}\left(R_{n}\right)$, is again smooth and proper and its special fibre is $X \times X^{\prime}$.

Set $\mathcal{X}_{\infty}:=\mathcal{X}, \mathcal{X}_{\infty}^{\prime}:=\mathcal{X}^{\prime}$, and $R_{\infty}:=R$ (notice that $\mathrm{D}_{\text {perf }}\left(\mathcal{X} \times_{R} \mathcal{X}^{\prime}\right) \simeq$ $\left.\mathrm{D}^{\mathrm{b}}\left(\mathcal{X} \times_{R} X^{\prime}\right)\right)$.

Proposition 2.12. Let $\varepsilon_{n} \in \mathrm{D}_{\text {perf }}\left(\mathcal{X}_{n} \times_{R_{n}} \mathcal{X}_{n}^{\prime}\right)$, with $n \in \mathbb{N} \cup\{\infty\}$, be such that its restriction $\mathcal{E}_{0}:=L j_{n}^{*} \varepsilon_{n}$ to the special fibre $X \times X^{\prime}$ is the kernel of a FourierMukai equivalence $\Phi_{\varepsilon_{0}}: \mathrm{D}^{\mathrm{b}}(X) \stackrel{\sim}{\longrightarrow} \mathrm{D}^{\mathrm{b}}\left(X^{\prime}\right)$. Then the Fourier-Mukai transform $\Phi_{\varepsilon_{n}}: \mathrm{D}_{\text {perf }}\left(X_{n}\right) \rightarrow \mathrm{D}_{\text {perf }}\left(X_{n}^{\prime}\right)$ is an equivalence.

Proof. It suffices to show that in both cases left and right adjoint functors are quasiinverse. Complete the trace morphism to a distinguished triangle

$$
\left(\varepsilon_{n}\right)_{\mathrm{L}} * \mathcal{E}_{n} \stackrel{\operatorname{tr} x_{n}}{\longrightarrow} \mathcal{O}_{\Delta x_{n}} \longrightarrow \mathcal{E}_{n} .
$$

Restricting it to the special fibre yields the distinguished triangle

$$
\left(\varepsilon_{0}\right)_{\mathrm{L}} * \mathcal{E}_{0} \stackrel{\operatorname{tr} X}{\longrightarrow} \mathcal{O}_{\Delta_{X}} \longrightarrow \mathscr{E}_{0} .
$$

(Use that the pull-back of the trace is the trace. Also the restriction of $\left(\mathcal{E}_{n}\right)_{\mathrm{L}}$ yields the kernel of the left adjoint of the restriction $\varepsilon_{0}$.)

As by assumption $\Phi_{\varepsilon_{0}}: \mathrm{D}^{\mathrm{b}}(X) \rightarrow \mathrm{D}^{\mathrm{b}}\left(X^{\prime}\right)$ defines an equivalence, the cone $\mathscr{E}_{0}$ is trivial. Thus, $g_{n} \in \mathrm{D}^{\mathrm{b}}\left(\mathcal{X}_{n} \times_{R_{n}} X_{n}^{\prime}\right)$ has trivial restriction to the special fibre $X \times X^{\prime}$ and, therefore, $\mathscr{E}_{n} \simeq 0$. This shows that $\operatorname{tr} x_{n}$ is an isomorphism. A similar argument proves that $\operatorname{tr}_{X_{n}^{\prime}}$ is an isomorphism for the case of the right adjoint.

Under the assumptions of the previous proposition, the same proof also yields an equivalence $\Phi_{\mathcal{E}_{n}}: \mathrm{D}^{\mathrm{b}}\left(\mathcal{X}_{n}\right) \rightarrow \mathrm{D}^{\mathrm{b}}\left(\mathcal{X}_{n}^{\prime}\right)$. 
Corollary 2.13. Let $\mathcal{E} \in \mathrm{D}^{\mathrm{b}}\left(\mathcal{X} \times_{R} X^{\prime}\right)$, such that $\Phi_{\mathcal{E}_{0}}: \mathrm{D}^{\mathrm{b}}(X) \stackrel{\sim}{\longrightarrow} \mathrm{D}^{\mathrm{b}}\left(X^{\prime}\right)$ is an equivalence. Then the Fourier-Mukai transform $\Phi_{\mathcal{E}_{K}}: \mathrm{D}^{\mathrm{b}}\left(\mathcal{X}_{K}\right) \stackrel{\sim}{\rightarrow} \mathrm{D}^{\mathrm{b}}\left(X_{K}^{\prime}\right)$ is an equivalence, where $\mathcal{E}_{K}$ denotes the image of $\mathcal{E}$ in $\mathrm{D}^{\mathrm{b}}\left(\left(\mathcal{X} \times_{R} \mathcal{X}^{\prime}\right)_{K}\right)$.

Proof. Indeed the inverse Fourier-Mukai functor $\Phi_{\mathcal{F}}:=\Phi_{\mathcal{E}}^{-1}: \mathrm{D}^{\mathrm{b}}\left(\mathcal{X}^{\prime}\right) \rightarrow \mathrm{D}^{\mathrm{b}}(\mathcal{X})$, which exists due to Proposition 2.12, descends to a Fourier-Mukai transform (see Section 2.3)

$$
\Phi_{\mathscr{F}_{K}}: \mathrm{D}^{\mathrm{b}}\left(X_{K}^{\prime}\right) \rightarrow \mathrm{D}^{\mathrm{b}}\left(\mathcal{X}_{K}\right),
$$

which clearly is an inverse to $\Phi_{\mathcal{E}_{K}}$.

\section{Properties of the derived category of the general fibre}

In this section we conclude the proof of Theorem 1.1. However, for most of the results it is enough to assume that $X$ is a smooth projective variety. More precisely, the assumption that $X$ is a surface with trivial canonical bundle is needed for the first time in Proposition 3.10.

In particular, we prove that $\mathrm{D}^{\mathrm{b}}\left(\mathcal{X}_{K}\right)$ is indeed equivalent to the derived category of $\operatorname{Coh}\left(X_{K}\right)$ and we study the Serre functor of $\mathrm{D}^{\mathrm{b}}\left(\mathcal{X}_{K}\right)$.

3.1. Comparing Hom-spaces. Let us now consider the pull-back under the closed embedding $\iota_{n}: X_{n} \hookrightarrow X$ which is a right exact functor $\iota_{n}^{*}: \operatorname{Coh}(X) \rightarrow \operatorname{Coh}\left(X_{n}\right)$ compatible with the $R$-linear respectively $R_{n}$-linear structure of the two categories. Its left derived functor

$$
L \iota_{n}^{*}: \mathrm{D}^{\mathrm{b}}(X) \longrightarrow \mathrm{D}_{\text {perf }}\left(\mathcal{X}_{n}\right)
$$

takes bounded complexes to perfect complexes (see Remark 2.4). When the derived context is clear, we will often simply write $\iota_{n}^{*}$ instead of $L \iota_{n}^{*}$. For $E \in \mathrm{D}^{\mathrm{b}}(\mathcal{X})$ one writes

$$
E_{n}:=\iota_{n}^{*} E=L \iota_{n}^{*} E \in \mathrm{D}^{\mathrm{b}}\left(X_{n}\right) .
$$

In particular, $E_{0}$ denotes the restriction of a complex $E$ on $X$ to the special fibre $X$. Clearly, $E \in \mathrm{D}^{\mathrm{b}}(\mathcal{X})$ is trivial if $E_{0} \simeq 0$.

One needs to be careful with the pull-back under $i_{n}: X_{n} \hookrightarrow X_{n+1}$, whose left derived functor $i_{n}^{*}: \mathrm{D}_{\text {perf }}\left(\mathcal{X}_{n+1}\right) \rightarrow \mathrm{D}_{\text {perf }}\left(\mathcal{X}_{n}\right)$ is well-defined for perfect complexes but not for bounded ones (see Remark 2.4).

Lemma 3.1. i) For $E, E^{\prime} \in \mathrm{D}^{\mathrm{b}}(\mathcal{X})$ there exists a functorial isomorphism

$$
R \operatorname{Hom}_{\mathrm{D}^{\mathrm{b}}(X)}\left(E, E^{\prime}\right) \otimes_{R}^{L} R_{n} \stackrel{\sim}{\longrightarrow} R \operatorname{Hom}_{\mathrm{D}_{\text {perf }}\left(X_{n}\right)}\left(L \iota_{n}^{*} E, L \iota_{n}^{*} E^{\prime}\right) .
$$


ii) For $m<n$ and $E, E^{\prime} \in \mathrm{D}_{\text {perf }}\left(\mathcal{X}_{n}\right)$ there exists a functorial isomorphism

$$
R \operatorname{Hom}_{D_{\text {perf }}\left(\chi_{n}\right)}\left(E, E^{\prime}\right) \otimes_{R_{n}}^{L} R_{m} \stackrel{\sim}{\longrightarrow} R \operatorname{Hom}_{D_{\text {perf }}\left(X_{m}\right)}\left(L i_{m, n}^{*} E, L i_{m, n}^{*} E^{\prime}\right) .
$$

Proof. The proofs of i) and ii) are identical. We just consider the first case.

Since we continue to work under the simplifying assumption that $X \rightarrow \operatorname{Spf}(R)$ is smooth and proper, the derived local Hom's are functors $R \mathscr{H o m}_{X}: \mathrm{D}^{\mathrm{b}}(\mathcal{X})^{\mathrm{op}} \times$ $\mathrm{D}^{\mathrm{b}}(\mathcal{X}) \rightarrow \mathrm{D}^{\mathrm{b}}(\mathcal{X})$. Also, by definition $(-) \otimes_{R}^{L} R_{n}$ is

$$
L \iota_{n}^{*}: \mathrm{D}^{\mathrm{b}}(\operatorname{Spf}(R)) \rightarrow \mathrm{D}_{\text {perf }}\left(\operatorname{Spec}\left(R_{n}\right)\right),
$$

the derived pull-back of the inclusion $\iota_{n}: \operatorname{Spec}\left(R_{n}\right) \hookrightarrow \operatorname{Spec}(R)$.

Thus the assumptions of [10, Proposition 7.1.2] are satisfied and we therefore have a functorial isomorphism

$$
L \iota_{n}^{*} R \operatorname{Hom}_{\chi}\left(E, E^{\prime}\right) \stackrel{\sim}{\longrightarrow} R \mathscr{H o m}_{\mathcal{X}_{n}}\left(L \iota_{n}^{*} E, L \iota_{n}^{*} E^{\prime}\right) .
$$

Further, applying the global section functor $R \Gamma \chi_{n}:=R \Gamma\left(\mathcal{X}_{n},-\right): \mathrm{D}^{\mathrm{b}}\left(\mathcal{X}_{n}\right) \rightarrow$ $\mathrm{D}^{\mathrm{b}}\left(\operatorname{Spec}\left(R_{n}\right)\right)$ to both sides, one finds

$$
\begin{aligned}
& L \iota_{n}^{*} R \Gamma \times \operatorname{RHom}_{\mathcal{X}}\left(E, E^{\prime}\right) \stackrel{(*)}{\simeq} R \Gamma \mathcal{X}_{n} L \iota_{n}^{*} R \operatorname{Hom}_{\mathcal{X}}\left(E, E^{\prime}\right)
\end{aligned}
$$

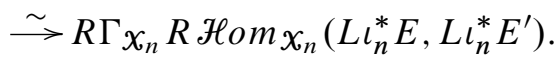

Together with $R \Gamma \circ R \mathscr{H o m}=R$ Hom, this proves the assertion.

Note that in $(*)$ we used the base change formula $L \iota_{n}^{*} \circ R \Gamma x \simeq R \Gamma \chi_{n} \circ L \iota_{n}^{*}$ which can be easily proved by adapting the argument of [12, Section 2.4]. More precisely, one could apply Kuznetsov's discussion to the cartesian triangle given by $\mathcal{X}_{n} \hookrightarrow X$ over $\operatorname{Spec}\left(R_{n}\right) \hookrightarrow \operatorname{Spf}(R)$. Corollary 2.23 in [12] shows that from the flatness of $\pi: X \rightarrow \operatorname{Spf}(R)$ one cannot only deduce the standard flat base change, but also the above assertion (see also [7, Chapter 3, Remark 3.33]). For flat base change in our more general context see [17].

The categories $\mathrm{D}^{\mathrm{b}}(X)$ and $\mathrm{D}^{\mathrm{b}}\left(\mathcal{X}_{K}\right)$ are $\mathbb{C}$-linear respectively $K$-linear triangulated categories with finite-dimensional Hom-spaces. The following numerical invariants turn out to be useful and well-behaved. For $E_{0}, E_{0}^{\prime} \in \mathrm{D}^{\mathrm{b}}(X)$ one sets:

$$
\chi_{0}\left(E_{0}, E_{0}^{\prime}\right):=\sum(-1)^{i} \operatorname{dim}_{\mathbb{C}} \operatorname{Ext}_{X}^{i}\left(E_{0}, E_{0}^{\prime}\right)
$$

and analogously for $E_{K}, E_{K}^{\prime} \in \mathrm{D}^{\mathrm{b}}\left(\mathcal{X}_{K}\right)$ :

$$
\chi_{K}\left(E_{K}, E_{K}^{\prime}\right):=\sum(-1)^{i} \operatorname{dim}_{K} \operatorname{Ext}_{K}^{i}\left(E_{K}, E_{K}^{\prime}\right) .
$$

As an immediate consequence of the discussion in Section 2.2, one finds the following two results which will be used in [9] to describe spherical and semi-rigid objects in $\mathrm{D}^{\mathrm{b}}\left(\mathcal{X}_{K}\right)$, when $X$ is a smooth projective $\mathrm{K} 3$ surface. 
Corollary 3.2. For any $E, E^{\prime} \in \mathrm{D}^{\mathrm{b}}(\mathcal{X})$ one has

$$
\chi_{0}\left(E_{0}, E_{0}^{\prime}\right)=\chi_{K}\left(E_{K}, E_{K}^{\prime}\right) .
$$

Proof. We have an isomorphism $R \operatorname{Hom}_{\mathrm{D}^{\mathrm{b}}(X)}\left(E_{0}, E_{0}^{\prime}\right) \simeq R \operatorname{Hom}_{\mathrm{D}^{\mathrm{b}}(\mathcal{X})}\left(E, E^{\prime}\right) \otimes^{L} \mathbb{C}$. This follows from Lemma $3.1 \mathrm{i}$ ). Since $R$ is a DVR, we have a decomposition of the $R$-module

$$
R \operatorname{Hom}_{\mathrm{D}^{\mathrm{b}}(\mathcal{X})}\left(E, E^{\prime}\right) \simeq R \operatorname{Hom}_{\mathrm{D}^{\mathrm{b}}(\mathcal{X})}\left(E, E^{\prime}\right)_{\text {free }} \oplus R \operatorname{Hom}_{\mathrm{D}^{\mathrm{b}}(\mathcal{X})}\left(E, E^{\prime}\right)_{\text {tor }}
$$

in its free and torsion part. Since for a torsion $R$-module $M$ one has $M \otimes^{L} \mathbb{C}=0$, this yields

$$
\chi_{0}\left(E_{0}, E_{0}^{\prime}\right)=\operatorname{dim}_{\mathbb{C}} R \operatorname{Hom}_{\mathrm{D}^{\mathrm{b}}(X)}\left(E_{0}, E_{0}^{\prime}\right)=\operatorname{dim}_{\mathbb{C}}\left(R \operatorname{Hom}_{\mathrm{D}^{\mathrm{b}}(X)}\left(E, E^{\prime}\right)_{\text {free }} \otimes \mathbb{C}\right) .
$$

On the other hand, by Proposition 2.9,

$$
\operatorname{dim}_{K} R \operatorname{Hom}_{\mathrm{D}^{\mathrm{b}}\left(\mathcal{X}_{K}\right)}\left(E_{K}, E_{K}^{\prime}\right)=\operatorname{dim}_{K}\left(R \operatorname{Hom}_{\mathrm{D}^{\mathrm{b}}(\mathcal{X})}\left(E, E^{\prime}\right)_{\text {free }} \otimes K\right) .
$$

This concludes the proof.

Of course, the single Hom-spaces could be quite different on the special and on the general fibre, but at least the standard semi-continuity result can be formulated in our setting.

Corollary 3.3. Let $E, E^{\prime} \in \mathrm{D}^{\mathrm{b}}(\mathcal{X})$. Then

$$
\operatorname{dim}_{\mathbb{C}} \operatorname{Hom}\left(E_{0}, E_{0}^{\prime}\right) \geq \operatorname{dim}_{K} \operatorname{Hom}_{K}\left(E_{K}, E_{K}^{\prime}\right) .
$$

Proof. We know that

$$
\operatorname{dim}_{K} \operatorname{Hom}_{\mathrm{D}^{\mathrm{b}}\left(X_{K}\right)}\left(E_{K}, E_{K}^{\prime}\right)=\mathrm{rk}_{R} \operatorname{Hom}_{\mathrm{D}^{\mathrm{b}}(\mathcal{X})}\left(E, E^{\prime}\right)_{\text {free }} .
$$

The conclusion follows from Lemma 3.1.

3.2. Serre functors. The relative canonical bundles $\omega_{\pi_{n}}:=\omega \mathcal{X}_{n} / R_{n}$ of $\pi_{n}: \mathcal{X}_{n} \rightarrow$ $\operatorname{Spec}\left(R_{n}\right)$ define a coherent sheaf $\omega_{\pi}$ on $\mathcal{X}$, the dualizing or canonical line bundle. The name is justified by the following observation (for more general statements see [1], [19]):

Lemma 3.4. Suppose $\pi: \mathcal{X} \rightarrow \operatorname{Spf}(R)$ is a smooth proper formal scheme of relative dimension $d$. Then there are functorial isomorphisms

$$
R \operatorname{Hom}_{\mathrm{D}^{\mathrm{b}}(\mathcal{X})}\left(E, \omega_{\pi}[d]\right) \stackrel{\sim}{\longrightarrow} R \operatorname{Hom}_{\mathrm{D}^{\mathrm{b}}(\operatorname{Spf}(R))}(R \Gamma x E, R),
$$

for all $E \in \mathrm{D}^{\mathrm{b}}(\mathcal{X})$. 
Proof. Notice that $\omega_{\pi_{n}}$ is the dualizing complex in $\mathrm{D}_{\text {perf }}\left(\mathcal{X}_{n}\right)$, that is

$$
R \operatorname{Hom}_{\mathrm{D}_{\text {perf }}\left(X_{n}\right)}\left(E_{n}, \omega_{\pi_{n}}[d]\right) \stackrel{\sim}{\longrightarrow} R \operatorname{Hom}_{\mathrm{D}_{\text {perf }}\left(R_{n}\right)}\left(R \Gamma \chi_{n} E_{n}, R_{n}\right),
$$

for any $E_{n} \in \mathrm{D}_{\text {perf }}\left(\mathcal{X}_{n}\right)$.

For any positive integer $n$, we have the following natural isomorphisms (using Lemma 3.1 twice)

$$
\begin{aligned}
R \operatorname{Hom}_{\mathrm{D}^{\mathrm{b}}(X)}\left(E, \omega_{\pi}[d]\right) \otimes_{R}^{L} R_{n} & \simeq R \operatorname{Hom}_{\mathrm{D}_{\text {perf }}\left(X_{n}\right)}\left(L \iota_{n}^{*} E, \omega_{\pi_{n}}[d]\right) \\
& \simeq R \operatorname{Hom}_{\mathrm{D}_{\mathrm{perf}}\left(R_{n}\right)}\left(R \Gamma \chi_{n} L \iota_{n}^{*} E, R_{n}\right) \\
& \simeq R \operatorname{Hom}_{\mathrm{D}^{\mathrm{b}}(\operatorname{Spf}(R))}(R \Gamma x E, R) \otimes_{R}^{L} R_{n} .
\end{aligned}
$$

(Notice that the last isomorphism uses again $L \iota_{n}^{*} \circ R \Gamma \mathcal{X} \simeq R \Gamma \mathcal{X}_{n} \circ L \iota_{n}^{*}$ as in the proof of Lemma 3.1.) Moreover, the resulting isomorphisms

$$
f_{n}: \operatorname{Hom}_{\mathrm{D}^{\mathrm{b}}(X)}\left(E, \omega_{\pi}[d]\right) \otimes_{R}^{L} R_{n} \stackrel{\sim}{\rightarrow} R \operatorname{Hom}_{\mathrm{D}^{\mathrm{b}}(\operatorname{Spf}(R))}(R \Gamma X E, R) \otimes_{R}^{L} R_{n}
$$

are compatible under pull-back, i.e. $\bar{f}_{n+1}:=f_{n+1} \otimes_{R}^{L} \mathrm{id}_{R_{n}}=f_{n}$.

Taking the projective limits allows us to conclude the proof. More precisely, one uses the following general argument: Suppose we are given complexes $K^{\bullet}, L^{\bullet} \in$ $\mathrm{D}^{\mathrm{b}}\left(R\right.$-Mod) and isomorphisms $f_{n}: K^{\bullet} \otimes R_{R}^{L} R_{n} \stackrel{\sim}{\sim} L^{\bullet} \otimes_{R}^{L} R_{n}$ in $\mathrm{D}^{\mathrm{b}}\left(R_{n}\right.$-Mod) compatible in the above sense. Replacing $K^{\bullet}$ and $L^{\bullet}$ by complexes of free $R$-modules, we can assume that the $f_{n}$ are morphisms of complexes. Again using the projectivity of the modules $K^{i}$ and $L^{i}$, we deduce from the compatibility of $f_{n}$ and $f_{n+1}$ the existence of a homotopy $k^{i}: K^{i} \otimes R_{n} \longrightarrow L^{i-1} \otimes R_{n}$ between $f_{n}$ and $\bar{f}_{n+1}$, i.e. $f_{n}^{i}-\bar{f}_{n+1}^{i}=k^{i+1} d_{K}^{i}+d_{L}^{i-1} k^{i}$. Lift $k^{i}$ to $h^{i}: K^{i} \otimes R_{n+1} \longrightarrow L^{i} \otimes R_{n+1}$ and replace $f_{n+1}$ by the homotopic one $f_{n+1}+h d_{K}+d_{L} h$. With this new definition one has $f_{n}=\bar{f}_{n+1}$ as morphism of complexes homotopic to he original one. Continuing in this way, one obtains a projective system of morphisms of complexes. The limit is then well defined and yields an isomorphism $K^{\bullet} \stackrel{\sim}{\sim} L^{\bullet}$.

The functoriality of the constructions is straightforward.

We are now ready to show that the derived category of the general fibre, which is a $K$-linear category, has a Serre functor in the usual sense. The following proposition, saying that Serre duality holds true in $\mathrm{D}^{\mathrm{b}}\left(X_{K}\right)$, shows the advantage of working with $\mathrm{D}^{\mathrm{b}}\left(\mathcal{X}_{K}\right)$. The canonical bundle of the general fibre is by definition $\omega_{X_{K}}:=\left(\omega_{\pi}\right)_{K} \in$ $\operatorname{Coh}\left(\mathcal{X}_{K}\right)$

Proposition 3.5. Suppose $\pi: \mathcal{X} \rightarrow \operatorname{Spf}(R)$ is a smooth proper formal scheme of relative dimension $d$. Then the functor $E \longmapsto E \otimes \omega x_{K}[d]$ is a Serre functor for the $K$-linear category $\mathrm{D}^{\mathrm{b}}\left(\mathcal{X}_{K}\right)$, i.e. there are natural isomorphisms

$$
\operatorname{Hom}_{\mathrm{D}^{\mathrm{b}}\left(\mathcal{X}_{K}\right)}\left(E_{K}, E_{K}^{\prime}\right) \stackrel{\sim}{\rightarrow}\left(\operatorname{Hom}_{\mathrm{D}^{\mathrm{b}}\left(\mathcal{X}_{K}\right)}\left(E_{K}^{\prime}, E_{K} \otimes \omega x_{K}[d]\right)\right)^{*},
$$


for all $E_{K}, E_{K}^{\prime} \in \mathrm{D}^{\mathrm{b}}\left(\mathcal{X}_{K}\right)$, where $(-)^{*}$ denotes the dual $K$-vector space.

Proof. We follow the proof of [3, Proposition 5.1.1]. Let $E \in \mathrm{D}^{\mathrm{b}}(\mathcal{X})$ and let $E_{K} \in$ $\mathrm{D}^{\mathrm{b}}\left(\mathcal{X}_{K}\right)$ be its image under the natural projection. We have

$$
\begin{aligned}
& \operatorname{Hom}_{\mathrm{D}^{\mathrm{b}}\left(X_{K}\right)}\left(E_{K}, \omega x_{K}[d]\right) \simeq \operatorname{Hom}_{\mathrm{D}^{\mathrm{b}}(\mathcal{X})}\left(E, \omega_{\pi}[d]\right) \otimes_{R} K \\
& \simeq \operatorname{Hom}_{\mathrm{D}^{\mathrm{b}}(\operatorname{Spf}(R))}(R \Gamma x E, R) \otimes_{R} K \\
& \simeq \operatorname{Hom}_{R}\left(\oplus_{s} R^{s} \Gamma x E[-s], R\right) \otimes_{R} K \\
& \simeq \operatorname{Hom}_{R}\left(R^{0} \Gamma_{\chi} E, R\right) \otimes_{R} K \\
& \simeq \operatorname{Hom}_{R}\left(\operatorname{Hom}_{\mathrm{D}^{\mathrm{b}}(\mathcal{X})}\left(\mathcal{O}_{X}, E\right), R\right) \otimes_{R} K \\
& \left.\simeq\left(\operatorname{Hom}_{\mathrm{D}^{\mathrm{b}}(X)}\left(\mathcal{O}_{X}, E\right) \otimes_{R} K\right)\right)^{*} \\
& \simeq\left(\operatorname{Hom}_{\mathrm{D}^{\mathrm{b}}\left(X_{K}\right)}\left(\mathcal{O}_{x_{K}}, E_{K}\right)\right)^{*},
\end{aligned}
$$

where the first and the last isomorphisms follow from Proposition 2.9, while the second is Lemma 3.4, and all the others are simple consequences of the fact that $R$ is a DVR. Dualizing (with respect to $K$ ) we have

$$
\operatorname{Hom}_{\mathrm{D}^{\mathrm{b}}\left(X_{K}\right)}\left(\mathcal{O}_{X_{K}}, E_{K}\right) \simeq\left(\operatorname{Hom}_{\mathrm{D}^{\mathrm{b}}\left(X_{K}\right)}\left(E_{K}, \omega \mathcal{X}_{K}[d]\right)\right)^{*} .
$$

Now, let $E, E^{\prime} \in \mathrm{D}^{\mathrm{b}}(\mathcal{X})$ and let $E_{K}, E_{K}^{\prime} \in \mathrm{D}^{\mathrm{b}}\left(\mathcal{X}_{K}\right)$ be their images. Since $E$ and $E^{\prime}$ are perfect complexes, the natural map

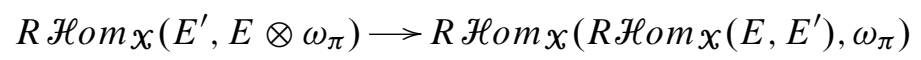

is an isomorphism. Indeed the statement is local and we can assume $E$ and $E^{\prime}$ be bounded complexes of free sheaves. In that case the claim is obvious.

Then one concludes by

$$
\begin{aligned}
& \operatorname{Hom}_{D^{\mathrm{b}}\left(X_{K}\right)}\left(E_{K}, E_{K}^{\prime}\right) \\
& \simeq \operatorname{Hom}_{\mathrm{D}^{\mathrm{b}}(\mathcal{X})}\left(E, E^{\prime}\right) \otimes_{R} K \\
& \simeq \operatorname{Hom}_{\mathrm{D}^{\mathrm{b}}(\mathcal{X})}\left(\mathcal{O}_{X}, R \operatorname{Hom}_{X}\left(E, E^{\prime}\right)\right) \otimes_{R} K \\
& \simeq\left(\operatorname{Hom}_{\mathrm{D}^{\mathrm{b}}(X)}\left(R \mathscr{H o m} \mathcal{X}\left(E, E^{\prime}\right), \omega_{\pi}[d]\right) \otimes_{R} K\right)^{*} \\
& \simeq\left(\operatorname{Hom}_{\mathrm{D}^{\mathrm{b}}(\mathcal{X})}\left(\mathcal{O}_{X}, R \operatorname{Hom}_{\chi}\left(R \mathscr{H o m}_{\chi}\left(E, E^{\prime}\right), \omega_{\pi}[d]\right)\right) \otimes_{R} K\right)^{*} \\
& \simeq\left(\operatorname{Hom}_{\mathrm{D}^{\mathrm{b}}(\mathcal{X})}\left(\mathcal{O}_{X}, R \operatorname{Hom}_{X}\left(E^{\prime}, E \otimes \omega_{\pi}[d]\right)\right) \otimes_{R} K\right)^{*} \\
& \simeq\left(\operatorname{Hom}_{\mathrm{D}^{\mathrm{b}}(X)}\left(E^{\prime}, E \otimes \omega_{\pi}[d]\right) \otimes_{R} K\right)^{*} \\
& \simeq\left(\operatorname{Hom}_{\mathrm{D}^{\mathrm{b}}\left(X_{K}\right)}\left(E_{K}^{\prime}, E_{K} \otimes \omega x_{K}[d]\right)\right)^{*},
\end{aligned}
$$

where the first and the last isomorphisms follow from Proposition 2.9 while the third is Lemma 3.4. The functoriality is clear.

If $X$ is a smooth projective surface with trivial canonical bundle, then $\omega_{\pi}$ is trivial and thus $\omega x_{K}$ is trivial as well. Therefore, in this case, the Serre functor of $\mathrm{D}^{\mathrm{b}}\left(\mathcal{X}_{K}\right)$ is isomorphic to the square of the shift functor and $\mathrm{D}^{\mathrm{b}}\left(\mathcal{X}_{K}\right)$ is thus a $\mathrm{K} 3$ (or 2-Calabi-Yau) category as claimed in Theorem 1.1. 
3.3. A technical result. Instead of taking Verdier quotients of derived categories, one could also consider derived categories of Serre quotients of the underlying abelian categories. Let us start with a few observations that should hold for the more general situation of the natural projection $\mathrm{D}^{\mathrm{b}}(\mathscr{B}) \rightarrow \mathrm{D}^{\mathrm{b}}(\mathscr{B} / \mathcal{A})$ induced by the quotient of a (non localizing) Serre subcategory $\mathcal{A} \subset \mathscr{B}$ of an abelian category $\mathscr{B}$. We could not find a good reference for the general case and since the proofs are technically easier, we restrict to the Serre subcategory $\operatorname{Coh}\left(X_{0}\right)_{0} \subset \operatorname{Coh}(X)$ with quotient $\operatorname{Coh}\left(x_{K}\right)$.

The following technical result is probably well-know in other contexts. It is the first step towards the proof of the existence of an exact equivalence $\mathrm{D}^{\mathrm{b}}\left(\mathcal{X}_{K}\right) \simeq$ $\mathrm{D}^{\mathrm{b}}\left(\boldsymbol{C o h}\left(\mathcal{X}_{K}\right)\right)$. We include the proof here for the convenience of the reader.

Proposition 3.6. The natural exact functor $Q: \mathrm{D}^{\mathrm{b}}(\operatorname{Coh}(\mathcal{X})) \rightarrow \mathrm{D}^{\mathrm{b}}\left(\mathbf{C o h}\left(\mathcal{X}_{K}\right)\right)$ induces an exact equivalence

$$
\mathrm{D}^{\mathrm{b}}\left(\mathcal{X}_{K}^{c}\right)=\mathrm{D}^{\mathrm{b}}(\operatorname{Coh}(\mathcal{X})) / \mathrm{D}_{0}^{\mathrm{b}}(\operatorname{Coh}(\mathcal{X})) \stackrel{\sim}{\sim} \mathrm{D}^{\mathrm{b}}\left(\operatorname{Coh}\left(\mathcal{X}_{K}\right)\right)
$$

For an abelian category $\mathbf{A}$, we denote by $\mathrm{C}^{\mathrm{b}}(\mathbf{A})$ the abelian category of bounded complexes of objects in $\mathbf{A}$ and by $\mathrm{K}^{\mathrm{b}}(\mathbf{A})$ the category of bounded complexes modulo homotopy.

Lemma 3.7. The natural projection $Q: \mathrm{D}^{\mathrm{b}}(\operatorname{Coh}(\mathcal{X})) \rightarrow \mathrm{D}^{\mathrm{b}}\left(\operatorname{Coh}\left(\mathcal{X}_{K}\right)\right)$ is essentially surjective.

Proof. Let $F^{\bullet}$ be a bounded complex in the quotient category $\mathrm{C}^{\mathrm{b}}\left(\operatorname{Coh}\left(\mathcal{X}_{K}\right)\right)$, i.e. $F^{i}=E_{K}^{i}$ for some $E^{i} \in \operatorname{Coh}(\mathcal{X})$ and differentials $d^{i} \in \operatorname{Hom}_{K}\left(F^{i}, F^{i+1}\right)=$ $\operatorname{Hom}\left(E^{i}, E^{i+1}\right) \otimes K$ (see Proposition 2.3).

Suppose $F^{i}=0$ for $|i|>n$ for some $n>0$. Then there exists $N \gg 0$ such that $t^{N} d^{i} \in \operatorname{Hom}\left(E^{i}, E^{i+1}\right)$. Furthermore, we can choose $N$ large enough such that $\left(t^{N} d^{i+1}\right) \circ\left(t^{N} d^{i}\right)$ is trivial in $\operatorname{Coh}(X)$ for all $i$. Let $\widetilde{E}^{\bullet}$ be the complex with objects $\widetilde{E}^{i}=E^{i}$ and differentials $\tilde{d}^{i}:=t^{N} d^{i}$. Then $t^{N(n-i)}: \widetilde{E}_{K}^{i} \stackrel{\sim}{\longrightarrow} F^{i}$ defines an isomorphism of complexes $Q\left(\widetilde{E}^{\bullet}\right) \stackrel{\sim}{\longrightarrow} F^{\bullet}$.

Thus, in particular, in order to prove Proposition 3.6, that is, to demonstrate that the natural functor induces an equivalence $\mathrm{D}^{\mathrm{b}}\left(\mathcal{X}_{K}^{c}\right) \stackrel{\sim}{\rightarrow} \mathrm{D}^{\mathrm{b}}\left(\operatorname{Coh}\left(\mathcal{X}_{K}\right)\right)$, it re-

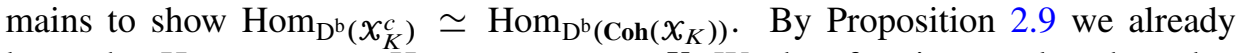
know that $\operatorname{Hom}_{\mathrm{D}^{\mathrm{b}}\left(X_{K}^{c}\right)} \simeq \operatorname{Hom}_{\mathrm{D}^{\mathrm{b}}(\operatorname{Coh}(X))} \otimes K$. We therefore just need to show that $\mathrm{D}^{\mathrm{b}}\left(\mathcal{X}_{K}^{c}\right) \rightarrow \mathrm{D}^{\mathrm{b}}\left(\mathbf{C o h}\left(\mathcal{X}_{K}\right)\right)$ induces as well isomorphisms $\operatorname{Hom}_{\mathrm{D}^{\mathrm{b}}(\operatorname{Coh}(X))} \otimes K \simeq$ $\operatorname{Hom}_{\mathrm{D}^{\mathrm{b}}\left(\operatorname{Coh}\left(X_{K}\right)\right)}$. This will be the content of Lemma 3.9.

In the following we shall frequently use the much easier fact that

$$
\operatorname{Hom}_{\mathrm{C}^{\mathrm{b}}(\operatorname{Coh}(x))}\left(E_{1}^{\bullet}, E_{2}^{\bullet}\right) \otimes K \simeq \operatorname{Hom}_{\mathrm{C}^{\mathrm{b}}\left(\operatorname{Coh}\left(x_{K}\right)\right)}\left(Q\left(E_{1}^{\bullet}\right), Q\left(E_{2}^{\bullet}\right)\right),
$$


which is proved by the same argument as Proposition 2.3. One only has to observe in addition that in order to lift a morphism of complexes $f^{\bullet}: Q\left(E_{1}^{\bullet}\right) \longrightarrow Q\left(E_{2}^{\bullet}\right)$, one first lifts all $t^{n} f^{i}$ to $\tilde{f}^{i}: E_{1}^{i} \longrightarrow E_{2}^{i}$ for some $n \gg 0$ and to make $\tilde{f}^{\bullet}$ a map of complexes on $\mathcal{X}$ one might have to annihilate kernel and cokernel of $d_{E_{2}}^{i} \circ \tilde{f}^{i}-$ $\tilde{f}^{i+1} \circ d_{E_{1}}^{i}$ by multiplying with yet another high power of $t$.

Lemma 3.8. Let $E_{1}^{\bullet}, E_{2}^{\bullet} \in \mathrm{C}^{\mathrm{b}}(\operatorname{Coh}(\mathcal{X}))$ and let $h \in \operatorname{Hom}_{\mathrm{C}^{\mathrm{b}}(\operatorname{Coh}(\mathcal{X}))}\left(E_{1}^{\bullet}, E_{2}^{\bullet}\right)$ be such that $Q(h)$ is a quasi-isomorphism in $\mathrm{C}^{\mathrm{b}}\left(\mathbf{C o h}\left(\mathcal{X}_{K}\right)\right)$. Then there exist two complexes $F_{1}^{\bullet}, F_{2}^{\bullet}$ and two morphisms $f_{1}: F_{1}^{\bullet} \rightarrow E_{1}^{\bullet}, f_{2}: F_{2}^{\bullet} \rightarrow E_{2}^{\bullet}$ in $\mathrm{C}^{\mathrm{b}}(\mathbf{C o h}(\mathcal{X}))$ such that $Q\left(f_{1}\right)$ and $Q\left(f_{2}\right)$ are isomorphisms in $\mathrm{C}^{\mathrm{b}}\left(\mathbf{C o h}\left(\mathcal{X}_{K}\right)\right)$ and $Q\left(f_{2}\right)^{-1} \circ Q(h) \circ Q\left(f_{1}\right)=$ $Q(\gamma)$, with $\gamma$ a quasi-isomorphism in $\mathrm{C}^{\mathrm{b}}(\operatorname{Coh}(\mathcal{X}))$.

Proof. The proof is based on calculations similar to the ones in the proof of Lemma 3.7, we will therefore be brief. We shall outline a construction that yields a $\gamma$ inducing an isomorphism in the lowest cohomology and leave the higher cohomologies to the reader.

Up to shift, we can assume that $E_{1}^{\bullet}, E_{2}^{\bullet}$ and hence $h$ are concentrated in $[0, r]$. The induced maps $\mathscr{H}^{i}(h): \mathscr{H}^{i}\left(E_{1}^{\bullet}\right) \longrightarrow \mathscr{H}^{i}\left(E_{2}^{\bullet}\right)$ on cohomology have kernels and cokernels in $\operatorname{Coh}(\mathcal{X})_{0}$ since $Q(h)$ is a quasi-isomorphism in $\mathrm{C}^{\mathrm{b}}\left(\mathbf{C o h}\left(\mathcal{X}_{K}\right)\right)$,.

In the following discussion we use the observation that for any $K \in \operatorname{Coh}(\mathcal{X})$ and $n \gg 0$, the sheaf $t^{n} K$ is $R$-flat and the cokernel of the inclusion $t^{n} K \hookrightarrow K$ is an object of $\operatorname{Coh}(\mathcal{X})_{0}$ (see Section 2.1).

We first construct a complex $Z_{1,0}^{\bullet} \in \mathrm{C}^{\mathrm{b}}(\mathbf{C o h}(\mathcal{X}))$ and a morphism $f_{1,0}^{\prime}: Z_{1,0}^{\bullet} \rightarrow$ $E_{1}^{\bullet}$ such that $\operatorname{ker}\left(\mathscr{H}^{0}\left(h \circ f_{1,0}^{\prime}\right)\right)$ is trivial. If $n \gg 0$, then $Z_{1,0}^{0}:=t^{n} E_{1}^{0}$ is $R$-flat and the inclusion $i_{1,0}^{\prime}: Z_{1,0}^{0}:=t^{n} E_{1}^{0} \longrightarrow E_{1}^{0}$ is an isomorphism in $\operatorname{Coh}\left(\mathcal{X}_{K}\right)$. Then the map of complexes
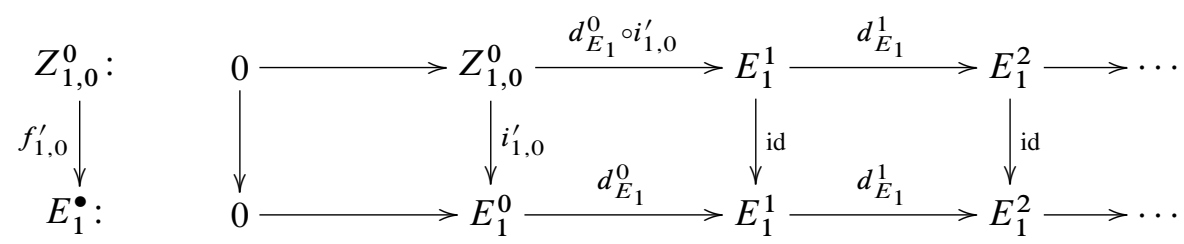

yields an isomorphism in $\mathrm{C}^{\mathrm{b}}\left(\boldsymbol{C o h}\left(\mathcal{X}_{K}\right)\right)$. As a subsheaf of the $R$-flat sheaf $Z_{1,0}^{0}$, the kernel $\operatorname{ker}\left(d_{E_{1}}^{0} \circ i_{1,0}^{\prime}\right)$ is also $R$-flat. Since $\operatorname{ker}\left(\mathscr{H}^{0}\left(h \circ f_{1,0}^{\prime}\right)\right) C \operatorname{ker}\left(d_{E_{1}}^{0} \circ i_{1,0}^{\prime}\right)$ and $\operatorname{ker}\left(\mathscr{H}^{0}\left(h \circ f_{1,0}^{\prime}\right)\right) \in \mathbf{C o h}(\mathcal{X})_{0}$, this implies $\operatorname{ker}\left(\mathscr{H}^{0}\left(h \circ f_{1,0}^{\prime}\right)=0\right.$. To simplify the notation, we assume henceforth $E_{1}^{\bullet}=Z_{1,0}^{\bullet}$ and $h=h \circ f_{1,0}^{\prime}$, i.e. that $\mathscr{H}^{0}(h)$ is injective.

Now we define two complexes $F_{1,0}^{\bullet}$ and $F_{2,0}^{\bullet}$ in $\mathrm{C}^{\mathrm{b}}(\boldsymbol{C o h}(\mathcal{X}))$ and morphisms $f_{1,0}: F_{1,0}^{\bullet} \rightarrow E_{1}^{\bullet}$ and $f_{2,0}: F_{2,0}^{\bullet} \rightarrow E_{2}^{\bullet}$ yielding isomorphisms in $\mathrm{C}^{\mathrm{b}}\left(\operatorname{Coh}\left(\mathcal{X}_{K}\right)\right)$, 
such that there exists a morphism $h_{0}: F_{1,0}^{\bullet} \longrightarrow F_{2,0}^{\bullet}$ with $h \circ f_{1,0}=f_{2,0} \circ h_{0}$ and $\operatorname{ker}\left(\mathscr{H}^{0}\left(h_{0}\right)\right)=\operatorname{Coker}\left(\mathscr{H}^{0}\left(h_{0}\right)\right)=0$.

To this end, consider the diagram

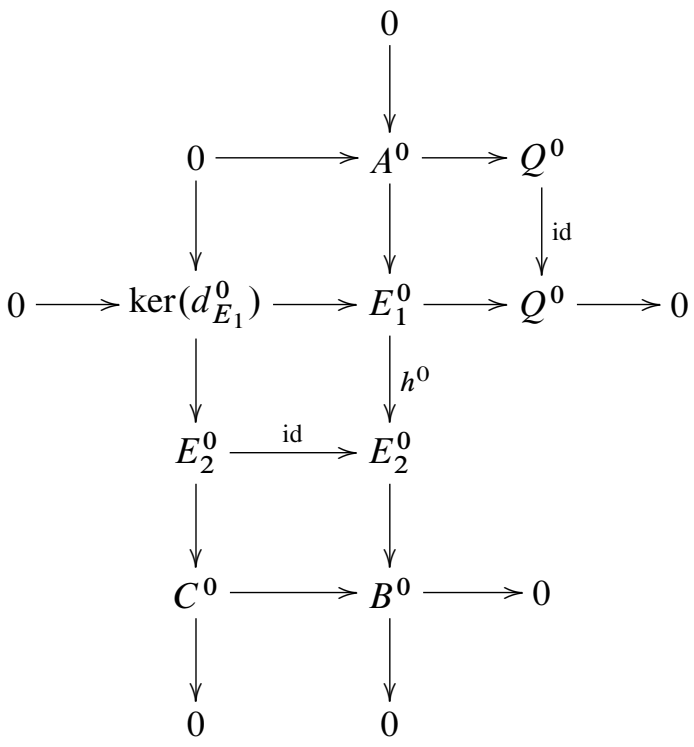

with exact rows and columns. The aim is to reduce to the case where $C^{0}$ is $R$-flat.

Let $D^{0}$ denote the cokernel of $A^{0} \longrightarrow Q^{0}$. Choose $n \gg 0$ and consider the short exact sequence $0 \longrightarrow D_{\text {flat }}^{0}:=t^{n} D^{0} \longrightarrow D^{0} \longrightarrow D_{\text {tor }}^{0} \longrightarrow 0$, where $D_{\text {tor }}^{0} \in \operatorname{Coh}(\mathcal{X})_{0}$ and $D_{\text {flat }}^{0}$ is $R$-flat, and define $F_{1,0}^{0}$ as the kernel of the composition $E_{1}^{0} \longrightarrow Q^{0} \longrightarrow D_{\text {tor }}^{0}$. By construction, the map of complexes

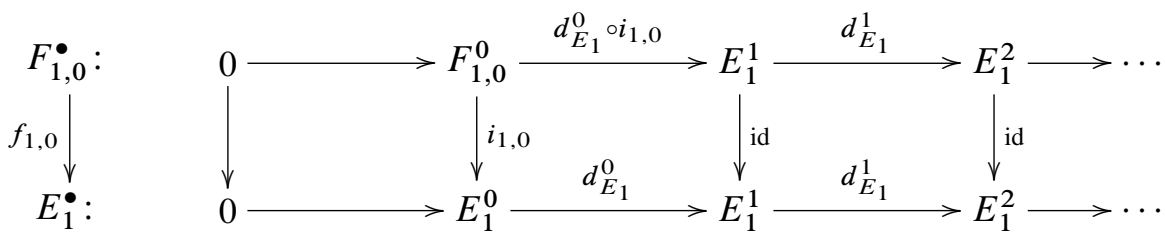

yields an isomorphism in $\mathrm{C}^{\mathrm{b}}\left(\mathbf{C o h}\left(\mathcal{X}_{K}\right)\right)$. Note that by construction the inclusion $A^{0} \longrightarrow E_{1}^{0}$ factorizes through $F_{1,0}^{0}$ and that the inclusion $\operatorname{ker}\left(d_{E_{1}}^{0}\right) \hookrightarrow E_{1}^{0}$ factorizes through $F_{1,0}^{0}$. Replace $E_{1}^{\bullet}$ by $F_{1,0}^{\bullet}$ and $h$ by $h \circ f_{1,0}$. Now, in the corresponding diagram (3.3) the inclusion $A^{0} \hookrightarrow Q^{0}$ has an $R$-flat cokernel.

Next, consider the exact sequence $0 \longrightarrow B_{\text {flat }}^{0} \longrightarrow B^{0} \longrightarrow B_{\text {tor }}^{0} \longrightarrow 0$ and define $F_{2,0}^{0}$ as the kernel of the composition $E_{2}^{0} \longrightarrow B^{0} \longrightarrow B_{\text {tor }}^{0}$, which natu- 
rally contains $\operatorname{im}\left(h^{0}\right)$. As before, the map of complexes

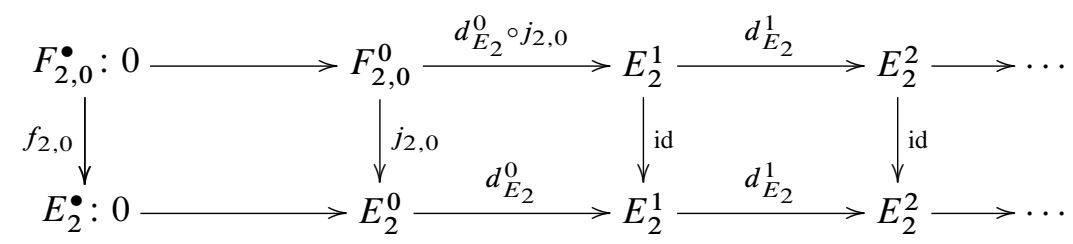

yields an isomorphism in $\mathrm{C}^{\mathrm{b}}\left(\mathbf{C o h}\left(\mathcal{X}_{K}\right)\right)$ and $h$ factorizes through $f_{2,0}$. Replace $E_{2}^{\bullet}$ by $F_{2,0}^{\bullet}$ and consider the corresponding diagram (3.3). Observe that now $C^{0}$ is $R$ flat (use the Snake Lemma). Since $\operatorname{Coker}\left(\mathscr{H}^{0}(h)\right)$ injects into $C^{0}$ and belongs to $\operatorname{Coh}(\mathcal{X})_{0}$, it must be trivial, as wanted.

In the spirit of Proposition 2.3 one can describe the homomorphisms in the derived category of the quotient as follows.

Lemma 3.9. For all complexes $E_{1}^{\bullet}, E_{2}^{\bullet} \in \mathrm{D}^{\mathrm{b}}(\operatorname{Coh}(\mathcal{X}))$ the natural exact functor $Q$ induces isomorphisms

$$
Q \otimes K: \operatorname{Hom}_{\mathrm{D}^{\mathrm{b}}(\operatorname{Coh}(\mathcal{X}))}\left(E_{1}^{\bullet}, E_{2}^{\bullet}\right) \otimes_{R} K \stackrel{\sim}{\rightarrow} \operatorname{Hom}_{\mathrm{D}^{\mathrm{b}}\left(\operatorname{Coh}\left(X_{K}\right)\right)}\left(Q\left(E_{1}^{\bullet}\right), Q\left(E_{2}^{\bullet}\right)\right) .
$$

Proof. We will prove the bijectivity of $Q \otimes K$ in two steps.

i) Injectivity. Let $f \in \operatorname{Hom}_{\mathrm{D}^{\mathrm{b}}(\operatorname{Coh}(X))}\left(E_{1}^{\bullet}, E_{2}^{\bullet}\right)$ such that $Q(f)=0$. By definition, $f$ may be represented by

$$
E_{1}^{\bullet} \stackrel{s_{0}}{\longleftarrow} F_{0}^{\bullet} \stackrel{g}{\longrightarrow} E_{2}^{\bullet},
$$

with $s_{0}$ a quasi-isomorphism in $\mathrm{C}^{\mathrm{b}}(\mathbf{C o h}(\mathcal{X}))$. Since $Q(f)=0$, there exists a commutative diagram in $\mathrm{K}^{\mathrm{b}}\left(\boldsymbol{C o h}\left(\mathcal{X}_{K}\right)\right)$ of the form

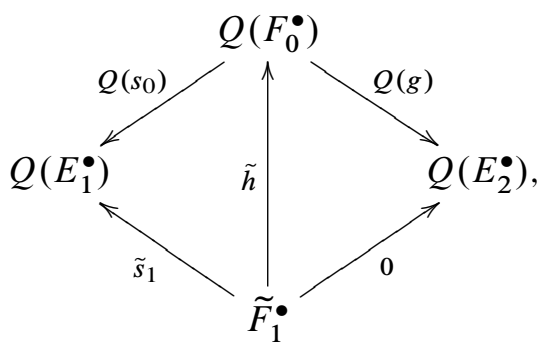

with $\tilde{s}_{1}$ and $\tilde{h}$ quasi-isomorphisms in $\mathrm{C}^{\mathrm{b}}\left(\boldsymbol{C o h}\left(\mathcal{X}_{K}\right)\right)$. By Lemma 3.7 and (3.2), we can assume that $\tilde{s}_{1}, \tilde{h}$, and $\tilde{F}_{1}^{\bullet}$ are in the image of $Q$, i.e. $\tilde{s}_{1}=Q\left(s_{1}\right), \tilde{h}=Q(h)$, and 
$\tilde{F}_{1}^{\bullet}=Q\left(F_{1}^{\bullet}\right)$. By Lemma 3.8 we have a commutative diagram in $\mathrm{K}^{\mathrm{b}}\left(\mathbf{C o h}\left(\mathcal{X}_{K}\right)\right)$

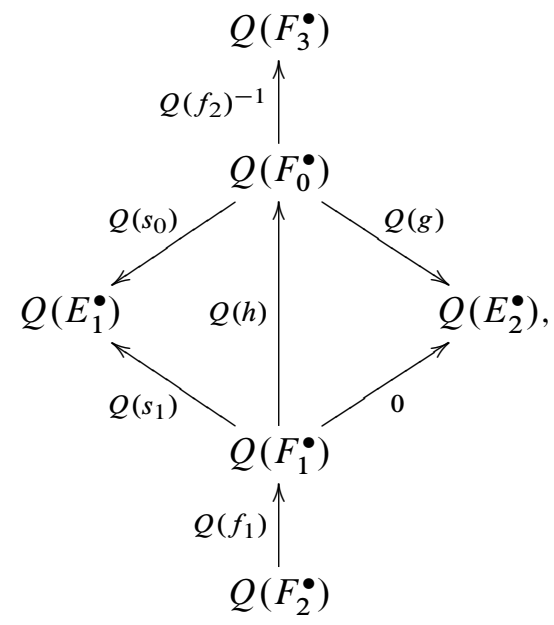

with $\gamma$ a quasi-isomorphism in $\mathrm{C}^{\mathrm{b}}(\mathbf{C o h}(\mathcal{X}))$ such that $Q(\gamma)=Q\left(f_{2}\right)^{-1} \circ Q(h) \circ$ $Q\left(f_{1}\right)$. So we have a commutative diagram in $\mathrm{K}^{\mathrm{b}}\left(\boldsymbol{C o h}\left(\mathcal{X}_{K}\right)\right)$

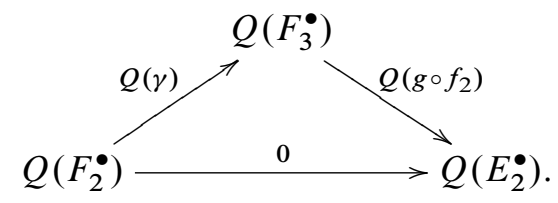

Hence, one finds $\tilde{k}^{i}: Q\left(F_{2}^{i}\right) \rightarrow Q\left(E_{2}^{i-1}\right)$ in $\operatorname{Coh}\left(\mathcal{X}_{K}\right)$ such that

$$
d_{Q\left(E_{2}\right)} \circ \tilde{k}+\tilde{k} \circ d_{Q\left(F_{2}\right)}-Q\left(g \circ f_{2} \circ \gamma\right)=0
$$

in $\operatorname{Coh}\left(X_{K}\right)$. By Proposition 2.3, there exists $N \gg 0$ such that $t^{N} \tilde{k}=Q(k)$ and

$$
d_{E_{2}} \circ k+k \circ d_{F_{2}}-\left(\left(t^{N}\left(g \circ f_{2}\right)\right) \circ \gamma\right)=0
$$

in $\operatorname{Coh}(\mathcal{X})$. So $\left(t^{N}\left(g \circ f_{2}\right)\right) \circ \gamma=0$ in $\mathrm{K}^{\mathrm{b}}(\mathbf{C o h}(\mathcal{X}))$. Therefore, there is a quasiisomorphism $\gamma^{\prime}: E_{2}^{\bullet} \longrightarrow F_{4}^{\bullet}$ in $\mathrm{K}^{\mathrm{b}}(\mathbf{C o h}(\mathcal{X}))$ such that $\gamma^{\prime} \circ\left(t^{N}\left(g \circ f_{2}\right)\right)=0$.

Then by Lemma 3.8, saying in particular that $Q\left(f_{2}\right)$ is an isomorphism in $\mathrm{C}^{\mathrm{b}}\left(\operatorname{Coh}\left(\mathcal{X}_{K}\right)\right)$, and by (3.2), there exist $h \in \operatorname{Hom}_{\mathrm{C}^{\mathrm{b}}(\operatorname{Coh}(X))}\left(F_{0}^{\bullet}, F_{3}^{\bullet}\right)$ and $n \gg 0$, such that $t^{n} f_{2} \circ h=$ id and hence $\gamma^{\prime} \circ\left(t^{n+N} g\right)=\gamma^{\prime} \circ\left(t^{N}\left(g \circ f_{2}\right)\right) \circ\left(t^{n} h\right)=0$ in $\mathrm{K}^{\mathrm{b}}(\boldsymbol{C o h}(\mathcal{X}))$. Hence $\gamma^{\prime} \circ\left(t^{n+N} f\right)=0$ in $\mathrm{D}^{\mathrm{b}}(\boldsymbol{C o h}(\mathcal{X}))$. Since $\gamma^{\prime}$ is a quasiisomorphism, this yields $t^{n+N} f=0$ in $\mathrm{D}^{\mathrm{b}}(\mathbf{C o h}(\mathcal{X}))$.

ii) Surjectivity. Let $\tilde{f} \in \operatorname{Hom}_{\mathrm{D}^{\mathrm{b}}\left(\mathcal{X}_{K}\right)}\left(Q\left(E_{1}^{\bullet}\right), Q\left(E_{2}^{\bullet}\right)\right)$. Again by Lemma 3.7 and (3.2) we can assume that $\tilde{f}$ is of the form

$$
Q\left(E_{1}^{\bullet}\right)<Q\left(s_{0}\right) \quad Q\left(F_{0}^{\bullet}\right) \stackrel{Q(g)}{\longrightarrow} Q\left(E_{2}^{\bullet}\right) .
$$


Applying Lemma 3.8 to $Q\left(s_{0}\right)$ we get a commutative diagram in $\mathrm{C}^{\mathrm{b}}\left(\mathbf{C o h}\left(\mathcal{X}_{K}\right)\right)$ :

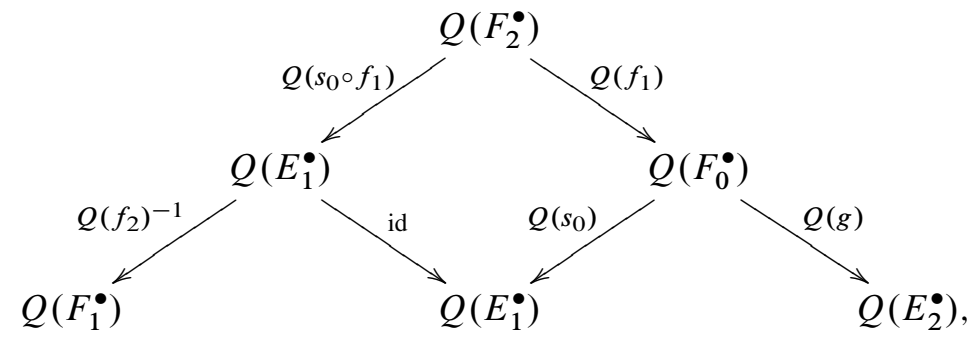

with $Q\left(f_{2}\right)^{-1} \circ Q\left(s_{0} \circ f_{1}\right)=Q(\gamma)$ and $\gamma$ a quasi-isomorphism, giving rise to a morphism $\alpha \in \operatorname{Hom}_{\mathrm{D}^{\mathrm{b}}(\mathbf{C o h}(\mathcal{X}))}\left(F_{1}^{\bullet}, E_{2}^{\bullet}\right)$ such that $Q(\alpha)$ is represented by (3.4).

If $\tilde{\beta} \in \operatorname{Hom}_{\mathrm{D}^{\mathrm{b}}\left(\mathbf{C o h}\left(X_{K}\right)\right)}\left(Q\left(F_{1}^{\bullet}\right), Q\left(E_{1}^{\bullet}\right)\right)$ corresponds to the diagram

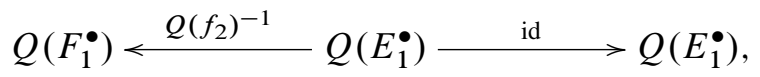

we have $\tilde{f} \circ \tilde{\beta}=Q(\alpha)$ and Hence $\tilde{f}=Q(\alpha) \circ \tilde{\beta}^{-1}$. Applying (3.2) to $Q\left(f_{2}\right)^{-1}$ one finds $n \gg 0$ and $g$, such that $t^{n} \tilde{\beta}^{-1}=Q\left(g_{2}\right)$. Thus $t^{n} \tilde{f}=Q(\alpha) \circ Q\left(g_{2}\right)$, as desired.

3.4. Back to the general fibre. In the definitions of $\mathrm{D}^{\mathrm{b}}\left(\mathcal{X}_{K}\right)$ and $\mathrm{D}^{\mathrm{b}}\left(\mathcal{X}_{K}^{c}\right)$ one divides by the categories $\mathrm{D}_{0}^{\mathrm{b}}(\mathcal{X})$ and $\mathrm{D}^{\mathrm{b}}\left(\operatorname{Coh}(\mathcal{X})_{0}\right)$ which, by Proposition 2.5 , are equivalent. The categories $\mathrm{D}_{\text {coh }}^{\mathrm{b}}\left(\mathcal{O}_{X}\right.$-Mod $)$ and $\mathrm{D}^{\mathrm{b}}(\operatorname{Coh}(\mathcal{X}))$ are in general not equivalent, so neither should be their quotients $\mathrm{D}^{\mathrm{b}}\left(\mathcal{X}_{K}^{c}\right)$ and $\mathrm{D}^{\mathrm{b}}\left(\mathcal{X}_{K}\right)$. However, for surfaces with trivial canonical bundle the situation is slightly better.

In the sequel we will write, by abuse of notation, $Q(E)=E_{K}$ where

$$
Q: \mathrm{D}^{\mathrm{b}}(\operatorname{Coh}(\mathcal{X})) \rightarrow \mathrm{D}^{\mathrm{b}}\left(\operatorname{Coh}\left(\mathcal{X}_{K}\right)\right)
$$

is defined as in Lemma 3.7.

Proposition 3.10. Suppose $\mathcal{X} \rightarrow \operatorname{Spf}(R)$ is a smooth proper formal scheme of dimension two with trivial canonical bundle, i.e. $\omega_{\pi} \simeq \mathcal{O}_{X}$. Then the natural exact functor

$$
\mathrm{D}^{\mathrm{b}}(\operatorname{Coh}(\mathcal{X})) \rightarrow \mathrm{D}^{\mathrm{b}}(\mathcal{X}) \rightarrow \mathrm{D}^{\mathrm{b}}\left(\mathcal{X}_{K}\right)
$$

induces an exact equivalence

$$
\begin{aligned}
& \mathrm{D}^{\mathrm{b}}\left(\operatorname{Coh}\left(X_{K}\right)\right) \stackrel{\sim}{\longrightarrow} \mathrm{D}^{\mathrm{b}}\left(\operatorname{Coh}\left(X_{0}\right)\right) / \mathrm{D}_{0}^{\mathrm{b}}(\operatorname{Coh}(X)) \stackrel{\sim}{\longrightarrow} \mathrm{D}^{\mathrm{b}}(\mathcal{X}) / \mathrm{D}_{0}^{\mathrm{b}}(\mathcal{X}) \\
& =\mathrm{D}^{\mathrm{b}}\left(\mathcal{X}_{K}^{c}\right) \quad=\mathrm{D}^{\mathrm{b}}\left(\mathcal{X}_{K}\right) .
\end{aligned}
$$


Proof. The first equivalence is the content of Proposition 3.6, so only the second equivalence needs a proof. By the universal property of localization and Remark 2.7 , the induced functor $\mathrm{D}^{\mathrm{b}}\left(\mathcal{X}_{K}^{c}\right) \rightarrow \mathrm{D}^{\mathrm{b}}\left(\mathcal{X}_{K}\right)$ exists. We need to prove it to be an equivalence.

Let us first show that it is fully faithful. Using induction on cohomologies, this would follow from

$$
\operatorname{Hom}_{\mathrm{D}^{\mathrm{b}}\left(X_{K}^{c}\right)}\left(E_{K}, F_{K}[i]\right) \stackrel{\sim}{\longrightarrow} \operatorname{Hom}_{\mathrm{D}^{\mathrm{b}}\left(X_{K}\right)}\left(E_{K}, F_{K}[i]\right)
$$

for all objects $E_{K}, F_{K} \in \operatorname{Coh}\left(\mathcal{X}_{K}\right)$ and all $i \in \mathbb{N}$. Here we use that the natural $K$-linear functor $\operatorname{Coh}\left(\mathcal{X}_{K}\right) \rightarrow \mathrm{D}^{\mathrm{b}}\left(\mathcal{X}_{K}\right)$, which by Propositions 2.3 and 2.9 is fully faithful, identifies $\operatorname{Coh}\left(\mathcal{X}_{K}\right)$ with the heart of a bounded $t$-structure on $\mathrm{D}^{\mathrm{b}}\left(\mathcal{X}_{K}\right)$ (see, e.g. [4, Lemma 3.2]).

In order to prove (3.5), we imitate the proof of [3, Proposition 5.2.1]. For fixed $F \in \operatorname{Coh}(\mathcal{X})$, write $\operatorname{Ext}_{I}^{*}(-, F)$ and $\operatorname{Ext}_{I I}^{*}(-, F)$ for the two contravariant $\delta$-functors $\operatorname{Ext}_{\mathrm{D}^{\mathrm{b}}(\operatorname{Coh}(\mathcal{X}))}^{*}(-, F) \otimes_{R} K$ and $\operatorname{Ext}_{\mathrm{D}^{\mathrm{b}}(\mathcal{X})}^{*}(-, F) \otimes_{R} K$ on $\operatorname{Coh}(\mathcal{X})$ with values in the category of $K$-vector spaces. They coincide in degree zero and $\operatorname{Ext}_{I}^{*}(-, F)$ is clearly universal. Thus, it suffices to prove that also $\operatorname{Ext}_{I I}^{*}(-, F)$ is universal. By Grothendieck's result (see [6, Theorem 1.3.A]), this would follow from $\operatorname{Ext}_{I I}^{i}(-, F)$ being coeffaceable for $i>0$. Recall that $\operatorname{Ext}_{I I}^{i}(-, F)$ is coeffaceable if for any $E \in \operatorname{Coh}(\mathcal{X})$, there exists an epimorphism $E^{\prime} \longrightarrow E$ in $\operatorname{Coh}(\mathcal{X})$ such that the induced map $\operatorname{Ext}_{I I}^{i}(E, F) \rightarrow \operatorname{Ext}_{I I}^{i}\left(E^{\prime}, F\right)$ is zero. Clearly $\operatorname{Ext}_{I}^{*}(-, F)$ is universal and $\operatorname{Ext}_{I}^{1}(E, F) \simeq \operatorname{Ext}_{I I}^{1}(E, F)$ (use that $\operatorname{Coh}\left(\mathcal{X}_{K}\right)$ is the heart of a bounded $t$ structure on $\mathrm{D}^{\mathrm{b}}\left(\mathcal{X}_{K}\right)$ and so the extensions in the abelian category coincides with those in the triangulated category). An easy modification of Grothendieck's original argument shows that it is enough to prove that $\operatorname{Ext}_{I I}^{i}(-, F)$ is coeffaceable for $i>1$. Moreover, by Proposition 3.5, we also have $\operatorname{Ext}_{I I}^{i}(E, F)=0$ for $i>2$. Hence we only have to show that $\operatorname{Ext}_{I I}^{2}(-, F)$ is effaceable.

By Lemma 3.11, for all rational sections $s$ of $\mathcal{X}$ over $R$, there exists a positive integer $n$ such that $\operatorname{Ext}_{I I}^{2}\left(\mathcal{M}_{s}^{n} E, F\right)=0$, where $\mathcal{M}_{s}$ denotes the ideal sheaf corresponding to $s$. Then, take $s$ and $s^{\prime}$ two disjoint rational $R$-sections of $\mathcal{X}$ and choose $n$ such that $\operatorname{Ext}_{I I}^{2}\left(\mathcal{M}_{s}^{n} E, F\right)=\operatorname{Ext}_{I I}^{2}\left(\mathcal{M}_{s^{\prime}}^{n} E, F\right)=0$. Since the canonical map $\mathcal{M}_{s}^{n} E \oplus \mathcal{M}_{s^{\prime}}^{n} E \longrightarrow E$ is surjective, we conclude by setting $E^{\prime}:=\mathcal{M}_{s}^{n} E \oplus \mathcal{M}_{s^{\prime}}^{n} E$.

Finally, one shows that $\mathrm{D}^{\mathrm{b}}\left(\mathcal{X}_{K}^{c}\right) \rightarrow \mathrm{D}^{\mathrm{b}}\left(\mathcal{X}_{K}\right)$ is also essentially surjective. Indeed, since $\operatorname{Coh}\left(\mathcal{X}_{K}\right)$ is in the natural way a heart of $t$-structures on both categories, this follows by induction over the length of complexes and the full faithfulness proved before.

Lemma 3.11. Let $E, F \in \operatorname{Coh}(\mathcal{X})$ and let s be a rational section of $\mathcal{X}$ over $R$ whose ideal sheaf in $\mathcal{O}_{X}$ is $\mathcal{M}_{s}$. Then there exists a positive integer $n$ such that

$$
\mathrm{Ext}_{\mathrm{D}^{\mathrm{b}}(\mathcal{X})}^{2}\left(\mathcal{M}_{s}^{n} E, F\right) \otimes_{R} K=0 .
$$


Proof. By Proposition 3.5 it suffices to show that one has $\operatorname{Hom}_{\mathrm{D}^{\mathrm{b}}(\mathcal{X})}\left(F, \mathcal{M}_{s}^{n} E\right) \otimes_{R}$ $K=0$ for $n \gg 0$. Since $\operatorname{Hom}_{\mathrm{D}^{\mathrm{b}}(\mathcal{X})}\left(F, \mathcal{M}_{s}^{n} E\right) \otimes_{R} K$ is finite dimensional over $K$, it is sufficient to show that, for $a \in \mathbb{N}$, there exists $b>a$ such that the natural inclusion $\operatorname{Hom}_{\mathrm{D}^{\mathrm{b}}(\mathcal{X})}\left(F, \mathcal{M}_{s}^{b} E\right) \otimes_{R} K \subset \operatorname{Hom}_{\mathrm{D}^{\mathrm{b}}(\mathcal{X})}\left(F, \mathcal{M}_{s}^{a} E\right) \otimes_{R} K$ is strict. (Use that $\left(\mathcal{M}_{s}^{b} E\right)_{K} \rightarrow\left(\mathcal{M}_{s}^{a} E\right)_{K}$ is still injective.)

Pick a non-zero $f \in \operatorname{Hom}_{\mathrm{D}^{\mathrm{b}}(\mathcal{X})}\left(F, \mathcal{M}_{s}^{a} E\right) \otimes_{R} K$. After multiplying with some power of $t$, we can assume $f \in \operatorname{Hom}_{\mathrm{D}^{\mathrm{b}}(\mathcal{X})}\left(F, \mathcal{M}_{s}^{a} E\right)$. Consider the exact sequence

$$
0 \longrightarrow E_{\mathrm{tor}} \rightarrow E \rightarrow E_{\mathrm{f}} \rightarrow 0,
$$

with $E_{\text {tor }} \in \operatorname{Coh}(X)_{0}$ and $E_{\mathrm{f}}$ flat over $R$. Consider the induced map $f: F \longrightarrow \mathcal{M}_{s}^{a} E_{\mathrm{f}}$. This is non-zero, since $f$ is not a torsion element. It is sufficient to show that there exists an integer $b>a$ such that $t^{n} f \notin \operatorname{Hom}_{\mathrm{D}^{\mathrm{b}}(\mathcal{X})}\left(F, \mathcal{M}_{s}^{b} E_{\mathrm{f}}\right)$, for all $n \in \mathbb{N}$. Thus, if $G:=\operatorname{Im}(f)$, it is enough to show that $G_{K} \subset\left(\mathcal{M}_{s}^{a} E_{\mathrm{f}}\right)_{K}$ is not contained in $\bigcap_{k}\left(\mathcal{M}_{s}^{k} E_{\mathrm{f}}\right)_{K}$.

Suppose to the contrary that $G_{K} \subset \bigcap\left(\mathcal{M}_{s}^{k} E_{\mathrm{f}}\right)_{K}$. We will show that then $G \subset$ $\bigcap \mathcal{M}_{s}^{k} E_{\mathrm{f}}$, which by the Krull Intersection Theorem would show $G=0$. Indeed, if $G \subset \mathcal{M}_{s}^{k} E_{\mathrm{f}}$, then also $G \subset \mathcal{M}_{s}^{k+1} E_{\mathrm{f}}$, as the induced map $G \longrightarrow\left(\mathcal{M}_{s}^{k} / \mathcal{M}_{s}^{k+1}\right) E_{\mathrm{f}}$ becomes the trivial map in $\operatorname{Coh}\left(\mathcal{X}_{K}\right)$, but $E_{\mathrm{f}}$ is $R$-flat and $t \notin \mathcal{M}_{s}$.

This applies to the case of $X$ a smooth complex projective surface with trivial canonical bundle and proves the last part of Theorem 1.1.

Remark 3.12. It should be possible to deduce from Proposition 3.10 that for smooth formal surfaces with trivial canonical bundle, $\mathrm{D}^{\mathrm{b}}(\mathbf{C o h}(X)) \stackrel{\sim}{\longrightarrow} \mathrm{D}_{\text {coh }}^{\mathrm{b}}\left(\mathcal{O}_{X}\right.$-Mod $) \simeq$ $\mathrm{D}^{\mathrm{b}}(\mathcal{X})$ is in fact an equivalence, but we shall not use this.

\section{Appendix. Verdier quotients and Serre subcategories}

This appendix collects known facts and definitions concerning quotients by Serre subcategories and Verdier quotients which were used throughout this paper. The main source we follow in the exposition is [13]. The reader is encouraged to look at Chapter 2 and Appendix A of [13] for a complete and readable account. Notice that we forget all set-theoretical issues which, in the case considered in the paper, do not give rise to problems (see [13, Section 2.2]).

A.1. Verdier quotients. Assume that $\mathbf{T}$ is a triangulated category with shift functor $\Sigma: \mathbf{T} \rightarrow \mathbf{T}$ (see [13, Chapter 1]). A full additive subcategory $\mathbf{D}$ is a triangulated subcategory if every object isomorphic to an object in $\mathbf{D}$ is in $\mathbf{D}$ and the inclusion functor $i: \mathbf{D} \longleftrightarrow \mathbf{T}$ is a triangulated functor with the additional requirement that, for any $D \in \mathbf{D}$ the isomorphism $\phi_{D}: i(\Sigma(D)) \longrightarrow \Sigma(i(D))$ is the identity. 
Definition A.1. A subcategory $\mathbf{D}$ of $\mathbf{T}$ is thick if it is triangulated and contains all direct summands of its objects.

If $\mathbf{D}$ is a triangulated subcategory of $\mathbf{T}$ one can form the Verdier quotient $\mathbf{T} / \mathbf{D}$ which is a triangulated category whose objects are the same as those of $\mathbf{T}$. To define the morphisms in T/D first consider the collection Mor $_{\mathbf{D}}$ of morphism $f: T_{1} \longrightarrow T_{2}$ in $\mathbf{T}$ sitting in an exact triangle

$$
T_{1} \stackrel{f}{\longrightarrow} T_{2} \longrightarrow Z \longrightarrow \Sigma\left(T_{1}\right)
$$

with $Z \in \mathbf{D}$. A morphism in $\mathbf{T} / \mathbf{D}$ between $T_{1}$ and $T_{2}$ is an equivalence class of diagrams

$$
\left(T_{1} \prec \stackrel{f}{\longleftarrow} T_{0} \stackrel{g}{\longrightarrow} T_{2}\right)
$$

with $f \in$ Mor $_{\mathbf{D}}$. We say that $\left(T_{1} \longleftarrow f_{1} T_{0} \stackrel{g_{1}}{\longrightarrow} T_{2}\right)$ and $\left(T_{1} \stackrel{f_{2}}{\longleftarrow} S_{0} \stackrel{g_{2}}{\longrightarrow} T_{2}\right.$ ) are equivalent if there is a third diagram $\left(T_{1} \stackrel{f_{3}}{\longleftarrow} Z_{0} \stackrel{g_{3}}{\longrightarrow} T_{2}\right)$ and morphisms $u: Z_{0} \longrightarrow T_{0}$ and $v: Z_{0} \longrightarrow S_{0}$ in Mor $_{\mathbf{D}}$ making the following diagram commutative

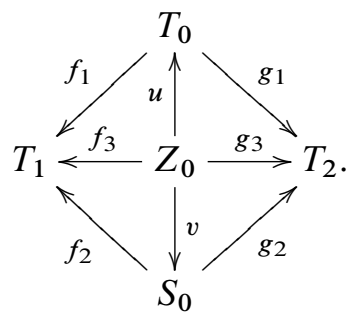

Roughly speaking, all morphisms in Mor $_{\mathbf{D}}$ become invertible.

Let $Q: \mathbf{T} \longrightarrow \mathbf{T} / \mathbf{D}$ be the natural triangulated functor which is called the Verdier localization. The kernel of $Q$ (i.e. the full additive subcategory of $\mathbf{T}$ consisting of objects mapped to zero by $Q$ ) is thick (see [13, Remark 2.1.7]). Hence, if $\mathbf{D}$ is thick, the kernel of $Q$ coincides with $\mathbf{D}$.

A.2. Serre subcategories. Let $\mathbf{A}$ be an abelian category and let $\mathbf{B} \subseteq \mathbf{A}$ be a full abelian subcategory. We say that $\mathbf{B}$ is thick if for $B_{1}, B_{2} \in \mathbf{B}$ and any short exact sequence

$$
0 \longrightarrow B_{1} \longrightarrow A \longrightarrow B_{2} \longrightarrow 0
$$

in $\mathbf{A}$, then $A$ belongs to $\mathbf{B}$ as well.

Definition A.2. A thick full subcategory $\mathbf{B}$ is a Serre subcategory of $\mathbf{A}$ if

i) Every object of $\mathbf{A}$ isomorphic to an object of $\mathbf{B}$ is in $\mathbf{B}$;

ii) Every quotient or subobject in $\mathbf{A}$ of an object in $\mathbf{B}$ is in $\mathbf{B}$. 
Given a Serre subcategory $\mathbf{B}$ of an abelian category $\mathbf{A}$ one can construct the quotient $\mathbf{A} / \mathbf{B}$ where the objects of $\mathbf{A} / \mathbf{B}$ are the same as those of $\mathbf{A}$. On the other hand, a morphism $A_{1} \longrightarrow A_{2}$ in $\mathbf{A} / \mathbf{B}$ is an equivalence class of diagrams

$$
\left(A_{1} \stackrel{s}{\longleftarrow} A_{0} \stackrel{t}{\longrightarrow} A_{2}\right)
$$

with $\operatorname{ker}(s), \operatorname{Coker}(s) \in \mathbf{B}$. The equivalence relation we mentioned has a definition which is analogue to the one explained in Section A.1 (see [13, Section A.2]).

A key fact is the following (see [13, Lemma A.2.3]):

Lemma A.3. The category $\mathbf{A} / \mathbf{B}$ is abelian. The natural functor $Q: \mathbf{A} \longrightarrow \mathbf{A} / \mathbf{B}$ is exact and takes object of $\mathbf{B}$ to objects in $\mathbf{A} / \mathbf{B}$ isomorphic to zero. Furthermore, $Q$ is universal with this property. The subcategory $\mathbf{B} \subseteq \mathbf{A}$ is the full subcategory of all objects $B \in \mathbf{A}$ such that $Q(A)$ is isomorphic to zero.

Acknowledgements. We thank the referee for many suggestions that improved the exposition of the paper. We gratefully acknowledge the support of the following institutions: Hausdorff Center for Mathematics, IHES, Imperial College, Istituto Nazionale di Alta Matematica, Max-Planck Institute, and SFB/TR 45. During the preparation of the final version of this paper the second named author was supported by NSF grant DMS-1001482.

\section{References}

[1] L. Alonso-Tarrío, A. Jeremías-López, and J. Lipman, Duality and flat base change on formal schemes. In Studies in duality on Noetherian formal schemes and non-Noetherian ordinary schemes, Contemp. Math. 244, Amer. Math. Soc., Providence, RI, 1999, 3-90; Corrigendum, Proc. Amer. Math. Soc. 131 (2003), no. 2, 351-357. Zbl 0953.14011 Zbl 1017.14007 MR 1716706 MR 1933323

[2] P. Berthelot, Cohomologie rigide et cohomologie rigide à support propre I. Prépublication IRMAR 96-03, 1996. http://perso.univ-rennes1.fr/pierre.berthelot/publis/Cohomologie_Rigide_I.pdf

[3] A. Bondal and M. Van den Bergh, Generators and representability of functors in commutative and noncommutative geometry. Moscow Math. J. 3 (2003), 1-36. Zbl 1135.18302 MR 1996800

[4] T. Bridgeland, Stability conditions on triangulated categories. Ann. of Math. 166 (2007), 317-346. Zbl 1137.18008 MR 2373143

[5] A. Căldăraru, The Mukai pairing, I: The Hochschild structure. arXiv:math/0308079v2 [math.AG]

[6] R. Hartshorne, Algebraic geometry. Grad. Texts in Math. 52, Springer-Verlag, New York 1977. Zbl 0367.14001 MR 0463157 
[7] D. Huybrechts, Fourier-Mukai transforms in algebraic geometry. Oxford Math. Monogr., Oxford University Press, Oxford, 2006. Zbl 1095.14002 MR 2244106

[8] D. Huybrechts, E. Macrì, and P. Stellari, Stability conditions for generic K3 surfaces. Compositio Math. 144 (2008), 134-162. Zbl 1152.14037 MR 2388559

[9] D. Huybrechts, E. Macrì, and P. Stellari, Derived equivalences of $K 3$ surfaces and orientation. Duke Math. J. 149 (2009), no. 3, 461-507. Zbl 05611496 MR 2553878

[10] L. Illusie, Généralités sur les conditions de finitude dans les catégories dérivées. In Séminaire de géométrie algébrique du Bois-Marie 1966/1967 (SGA 6), Lecture Notes in Math. 225, Springer-Verlag, Berlin 1971, 78-159. Zbl 0229.14009

[11] L. Illusie, Grothendieck's existence theorem in formal geometry. In Fundamenal algebraic geometry, Grothendieck's FGA explained, ed. by B. Fantechi et al., Math. Surveys Mongr. 123, Amer. Math. Soc., Providence, RI, 2005, 179-233. Zbl 1085.14001 MR 2223409

[12] A. Kuznetsov, Hyperplane sections and derived categories. Izv. Math. 70 (2006), 447-547. Zbl 1133.14016 MR 2238172

[13] A. Neeman, Triangulated categories. Ann. of Math. Stud. 148, Princeton University Press, Princeton, NJ, 2001. Zbl 0974.18008 MR 1812507

[14] D. Orlov, Equivalences of derived categories and K3 surfaces. J. Math. Sci. 84 (1997), 1361-1381. Zbl 0938.14019 MR 1465519

[15] M. Raynaud, Géométrie analytique rigide d'après Tate, Kiehl.... In Table ronde d'analyse non Archimédienne, Bull. Soc. Math. France Mém. 39-40, Société Mathématique de France, Paris, 1974, 319-327. Zbl 0299.14003 MR 0470254

[16] M. Rapoport and T. Zink, Period spaces for p-divisible groups. Ann. of Math. Stud. 141, Princeton University Press, Princeton, NJ, 1996. Zbl 0873.14039 MR 1393439

[17] N. Spaltenstein, Resolutions of unbounded complexes. Compositio Math. 65 (1988), 121-154. Zbl 0636.18006 MR 0932640

[18] B. Szendrói, Diffeomorphisms and families of Fourier-Mukai transforms in mirror symmetry. In Applications of algebraic geometry to coding theory, physics and computation, NATO Science Series II Math. Phys. Chem. 36, Kluwer, Dordrecht 2001, 317-337. Zbl 1017.14016 MR 1866907

[19] A. Yekutieli, Smooth formal embedings and the residue complex. Canad. J. Math. 50 (1998), 863-896. Zbl 0932.14007 MR 1638635

Received September 19, 2008

Daniel Huybrechts, Mathematisches Institut, Universität Bonn, Endenicher Allee 60, 53115 Bonn, Germany

E-mail: huybrech@math.uni-bonn.de

Emanuele Macrì, Mathematisches Institut, Universität Bonn, Endenicher Allee 60, 53115 Bonn, Germany

E-mail: macri@math.uni-bonn.de

Paolo Stellari, Dipartimento di Matematica "F. Enriques", Università degli Studi di Milano,

Via Cesare Saldini 50, 20133 Milano, Italy

E-mail: paolo.stellari@unimi.it 